\title{
Effective Algorithms for Solving Trace Minimization Problem in Multivariate Statistics
}

\author{
Jiao-fen Li (iD, Ya-qiong Wen, Xue-lin Zhou $(\mathbb{D}$, and Kai Wang \\ School of Mathematics and Computing Science, \\ Guangxi Colleges and Universities Key Laboratory of Data Analysis and Computation, \\ Guilin University of Electronic Technology, Guilin 541004, China \\ Correspondence should be addressed to Xue-lin Zhou; zhouxuelin0309@163.com
}

Received 4 April 2020; Revised 11 June 2020; Accepted 3 July 2020; Published 10 August 2020

Academic Editor: Hua Fan

Copyright (c) 2020 Jiao-fen Li et al. This is an open access article distributed under the Creative Commons Attribution License, which permits unrestricted use, distribution, and reproduction in any medium, provided the original work is properly cited.

\begin{abstract}
This paper develops two novel and fast Riemannian second-order approaches for solving a class of matrix trace minimization problems with orthogonality constraints, which is widely applied in multivariate statistical analysis. The existing majorization method is guaranteed to converge but its convergence rate is at best linear. A hybrid Riemannian Newton-type algorithm with both global and quadratic convergence is proposed firstly. A Riemannian trust-region method based on the proposed Newton method is further provided. Some numerical tests and application to the least squares fitting of the DEDICOM model and the orthonormal INDSCAL model are given to demonstrate the efficiency of the proposed methods. Comparisons with some latest Riemannian gradient-type methods and some existing Riemannian second-order algorithms in the MATLAB toolbox Manopt are also presented.
\end{abstract}

\section{Introduction}

Many multivariate statistical techniques are based on fitting a model to observed data. In most cases, these models are fitted by least squares, and as a consequence, one has to minimize a least squares loss function possibly subject to certain constraints on the parameters in the model. Least squares loss functions can often be expressed in terms of matrix trace functions. Sometimes, these have straightforward closed-form solutions, but also often such solutions are not available. In such cases, one may resort to iterative algorithms. The main purpose of the present paper is to provide several efficient algorithms that can be used for optimizing such matrix trace functions.

A class of functions that would include all those of interest in multivariate statistical analysis can probably not be given, but in practice many statistical problems can be reformulated as special cases of the optimization of the general function $[1-3]$ :

$$
c+\operatorname{tr}(A X)+\sum_{j=1}^{m} \operatorname{tr}\left(B_{j} X C_{j} X^{T}\right)
$$

where $A \in \mathbb{R}^{p \times n}(p \leq n), B_{j} \in \mathbb{R}^{n \times n}, C_{j} \in \mathbb{R}^{p \times p}, j=1, \ldots, m$; $X$ is an unknown $n \times p$ matrix, $c$ is a constant that does not depend on $X$, and $\operatorname{tr}(M)$ means the sum of the diagonal elements of $M$. The matrices $A, B_{j}$, and $C_{j}$ at this point are arbitrary but with the required orders. In fact, the problems of least squares fitting of a model $M_{X}$ (involving a matrix $X$ of model parameters) to a data matrix $Y$, that is, the minimization of $\sigma(X)=\left\|Y-M_{X}\right\|_{F}^{2}$ over $X$ (with $\|\cdot\|_{F}$ denoting the Frobenius norm), can often be written in a form like that of (1). To give an example, consider the case where the data matrix $Y$ has submatrices $Y_{k}$ and the model $M_{X}$ has submatrices of the form $F_{k} X G$; then,

$$
\begin{aligned}
\sigma(X)= & \left\|Y-M_{X}\right\|_{F}^{2}=\sum_{k=1}^{m}\left\|Y_{k}-F_{k} X G\right\|_{F}^{2} \\
= & \sum_{k=1}^{m} \operatorname{tr}\left(Y_{k}^{T} Y_{k}\right)-2 \sum_{k=1}^{m} \operatorname{tr}\left(G Y_{k}^{T} F_{k} X\right) \\
& +\sum_{k=1}^{m} \operatorname{tr}\left(G G^{T} X^{T} F_{k}^{T} F_{k} X\right) .
\end{aligned}
$$


Then, minimization of (2) is equivalent to minimization of (1) by letting $c=\sum_{k=1}^{m} \operatorname{tr}\left(Y_{k}^{T} Y_{k}\right), A=-2 \sum_{k=1}^{m} G Y_{k}^{T} F_{k}$, $B_{k}=F_{k}^{T} F_{k}$, and $C_{k}=G G^{T}, k=1, \ldots, m$.

The function $f(X)$ can be minimized over arbitrary $X$, but in many practical applications of multivariate statistics, constraints are imposed, the most frequent one being columnwise orthonormality: $X^{T} X=I_{p}[1-10]$, where $I_{p}$ denotes the identity matrix of size $p$. Another common constraint is that of reduced rank; that is, the rank of $X$ is forced to be less than or equal to $r(r \leq \min (n, p))$. The present paper discusses only the case of columnwise orthonormality. Therefore, the considered matrix trace minimization problem with constraints can be mathematically formulated by the following problem.

\section{Problem 1}

minimize $f(X)=c+\operatorname{tr}(A X)+\sum_{j=1}^{m} \operatorname{tr}\left(B_{j} X C_{j} X^{T}\right)$

subject to $\quad X \in \mathbb{R}^{n \times p}, X^{T} X=I_{p}$,

where $A \in \mathbb{R}^{p \times n}, B_{j} \in \mathbb{R}^{n \times n}$, and $C_{j} \in \mathbb{R}^{p \times p}, j=1, \ldots, m$ are given, $c$ is a constant, and $1 \leq p \leq n$.

Next, we present several practical applications of Problem 1 in multivariate statistics. The first example was proposed in $[1,4]$ for a least squares fit of a model for linear dynamical systems. Following their notation, let $B$ be a fixed $n \times n$ matrix, $D, F$, and $H$ be fixed $p \times p$ matrices, $X$ and $Y$ be fixed $n \times p$ matrices, and $Z$ be a variable $n \times p$. One of their subproblems is to minimize a function

$$
f_{1}(Z)=\left\|Z-B Z F^{T}-X D^{T}\right\|_{F}^{2}+\left\|Y-Z H^{T}\right\|_{F}^{2},
$$

over $Z$, subject to the constraint $Z^{T} Z=I_{p}$. This function can be expanded as

$$
\begin{aligned}
f_{1}(Z)= & c_{1}-2 \operatorname{tr}\left(D X^{T} Z\right)+2 \operatorname{tr}\left(F^{T} D X^{T} B Z\right) \\
& -2 \operatorname{tr}\left(H^{T} Y^{T} Z\right)+\operatorname{tr}\left(Z H^{T} H Z^{T}\right) \\
& +\operatorname{tr}\left(B^{T} B Z F^{T} F Z^{T}\right)-2 \operatorname{tr}\left(B Z F^{T} Z^{T}\right),
\end{aligned}
$$

where $c_{1}$ denotes a constant term not depending on $Z$. It is readily verified that $f_{1}(Z)$ is the special case of $f(X)$ with $X$ replaced by $Z, A=-2 D X^{T}+2 F^{T} D X^{T} B-2 H^{T} Y^{T}, B_{1}=I_{n}$, $B_{2}=B^{T} B, B_{3}=-2 B, C_{1}=H^{T} H, C_{2}=F^{T} F$, and $C_{3}=F^{T}$.

The second example is the least squares fitting of the "DEDICOM model" $[1,5]$, which can be written as minimizing

$$
f_{2}(X, R)=\left\|F-X R X^{T}\right\|_{F}^{2}
$$

where $F$ is an arbitrary fixed $n \times n$ matrix and $X$ and $R$ are variable matrices of orders $n \times r$ and $r \times r$, respectively, with $X$ columnwise orthonormal. More detailed description of this model will be given in Numerical Experiments section. Kiers et al. [6] have given an alternating least squares algorithm for fitting this model. The DEDICOM function, with $R$ considered fixed, can be rewritten as

$$
f_{2}(X)=c_{2}-2 \operatorname{tr}\left(F^{T} X R X^{T}\right)
$$

where $c_{2}$ is a constant not depending on $X$. This is a special case of $f(X)$ with $A=0, B_{1}=-2 F^{T}, C_{1}=R$.

The third example is to fit the "orthonormal INDSCAL model" (see [7], pp. 110, 118 and $[1,10]$ ). Let $S_{j}$ be a fixed symmetric matrix of order $n \times n, X$ be a variable matrix of order $n \times p$, and $D_{j}$ be a variable diagonal matrix of order $p$, $j=1, \ldots, m$. Fitting the orthonormal INDSCAL model is equivalent to minimizing the function

$$
f_{3}\left(X, D_{j}\right)=\sum_{j=1}^{m}\left\|S_{j}-X D_{j} X^{T}\right\|_{F}^{2},
$$

subject to $X^{T} X=I_{p}$. As in Berge [8], $f_{3}$ can be minimized by alternatingly updating $X$ and $D_{j}, j=1, \ldots, m$. Function $f_{3}$, with $D_{j}, j=1, \ldots, m$, considered fixed, can be rewritten as

$$
f_{3}(X)=c_{3}-2 \sum_{j=1}^{m} \operatorname{tr}\left(S_{j} X D_{j} X^{T}\right)
$$

where $c_{3}$ is a constant with respect to $X$. This is the particular case of $f(X)$ with $A=0, B_{j}=-2 S_{j}, C_{j}=D_{j}, j=1, \ldots, m$.

As a final example, we consider the simultaneous components analysis problem [3,9] of minimizing

$$
f_{4}\left(S, Q_{l}, \ldots, Q_{m}\right)=\sum_{j=1}^{m}\left\|Z_{j}-Z_{j} R_{j}^{-1} S Q_{j}^{T}\right\|_{F}^{2},
$$

over $Q_{j}$ and $S$, subject to $S^{T} S=I_{p}$. By means of an alternating least squares algorithm, they updated all parameter matrices in turn. Function $f_{4}$, with $Q_{j}, j=1, \ldots, m$, considered fixed, can be expanded as

$$
f_{4}(S)=c_{4}+\operatorname{tr}\left(\left(-2 \sum_{j} Q_{j}^{T}\right) S\right)+\sum_{j} \operatorname{tr}\left(R_{j}^{-1} S Q_{j}^{T} Q_{j} S^{T}\right),
$$

which, ignoring the constant $c_{4}$, is a special case of $f(X)$ with $X$ replaced by $S, A=\left(-2 \sum_{j} Q_{j}^{T}\right), B_{j}=R_{j}^{-1}$ and $C_{j}=Q_{j}^{T} Q_{j} j=1, \ldots, m$.

Concerning numerical approach for solving Problem 1, actually, Kiers et al. [1-3] have proposed the well-known majorization algorithm, which is based on majorizing $f(X)$ by a different function $g(X)$ whose minimum is easily obtained. The majorizing function is chosen such that the parameter set for which the majorizing function attains its minimum will also decrease the function to be minimized. For a more detailed description of such strategies, see [1-3]. However, since each iteration of the majorization method requires a full eigenvalue decomposition and the rate of convergence is at best linear, the method can potentially be very slow. An average of computational results of 10 random tests of the majorization method on Problem 1 is reported in Table 1, where the constant $c=0$ and $m=2$ and the given matrices $A, B_{1}, C_{1}, B_{2}$, and $C_{2}$ are all generated randomly by means of (in MATLAB style) $A=\operatorname{rand} n(p, n)$, $B_{1}=\operatorname{rand} n(n, n), C_{1}=\operatorname{rand} n(p, p), B_{2}=\operatorname{rand} n(n, n)$, and $C_{2}=\operatorname{rand} n(p, p)$. In Table 1, " $n, p$ ” means the dimension indexes and "CT." and "IT." mean the averaged total computing time in seconds and the averaged number of 
TABLE 1: Numerical results for the existing majorization method.

\begin{tabular}{lccccc}
\hline$n, p$ & CT. & IT. & Grad. & $f_{\text {diff }}$ & $X_{\text {feasi }}$ \\
\hline 30,10 & 0.599 & 1793 & $1.005 \times 10^{-4}$ & $1.561 \times 10^{-12}$ & $4.108 \times 10^{-15}$ \\
50,30 & 5.325 & 3595 & $1.001 \times 10^{-4}$ & $2.436 \times 10^{-13}$ & $1.221 \times 10^{-14}$ \\
100,50 & 51.804 & 3886 & $1.001 \times 10^{-4}$ & $8.030 \times 10^{-14}$ & $1.795 \times 10^{-14}$ \\
100,70 & 131.740 & 6792 & $1.000 \times 10^{-4}$ & $4.213 \times 10^{-14}$ & $2.231 \times 10^{-14}$ \\
200,50 & 333.110 & 4104 & $1.001 \times 10^{-4}$ & $4.545 \times 10^{-14}$ & $1.637 \times 10^{-14}$ \\
200,70 & 929.852 & 5318 & $1.000 \times 10^{-4}$ & $2.913 \times 10^{-14}$ & $2.232 \times 10^{-14}$ \\
\hline
\end{tabular}

iterations to achieve the stopping criteria, respectively. "Grad." means the averaged norm of Riemannian gradient grad $f\left(X_{k}\right)$, " $f_{\text {diff }}=\left|f\left(X_{k+1}\right)-f\left(X_{k}\right)\right|$ " means the averaged difference of the objective function values, and " $X_{\text {feasi }}$ " means the averaged value of feasibility $\left\|X_{k}^{T} X_{k}-I_{p}\right\|_{F}$. We can observe from Table 1 and the convergence histories of the objective function value that are unreported here that the existing majorization algorithm is a monotonically convergent algorithm for minimizing the matrix trace function iteratively. However, it requires a large number of iterations to converge to within a given tolerance because the rate of convergence of this method is at best linear.

To overcome the slow linear rate of convergence, in this work we reconsider Problem 1 under the framework of Riemannian optimization, by noting that the feasible set, St $(n, p)=\left\{X \in \mathbb{R}^{n \times p} \mid X^{T} X=I_{p}\right\}$, with $p \leq n$ is referred to the well-known Stiefel manifold, an $n p-(1 / 2) p(p+$ 1)-dimensional embedded submanifold of the vector space $\mathbb{R}^{n \times p}$. Riemannian optimization refers to optimization on Riemannian manifolds and has been extensively studied over decades. Optimization over the Stiefel manifold is an important special case of Riemannian optimization, which has recently aroused considerable research interests due to the wide applications in different fields such as the linear eigenvalue problem, the orthogonal Procrustes problem, the nearest low-rank correlation matrix problem, the Kohn-Sham total energy minimization, and singular value decomposition. Since optimization over the Stiefel manifold can be viewed as a general nonlinear optimization problem with constraints, many standard algorithms [11] in the Euclidean space can be generalized to manifold setting directly and have been explored and successfully applied to various applications, e.g., Riemannian steepest descent method [12], Riemannian curvilinear search method with Barzilai-Borwein (BB) steps [13], Riemannian Dai's nonmonotone-based conjugate gradient method $[14,15]$, Riemannian Polak-Ribière-Polyak-based nonlinear conjugate gradient method $[16,17]$, and Riemannian Fletcher-Reeves-based conjugate gradient method [18]. However, as we know, gradient-type algorithms often perform reasonably well but might converge slowly when the generated iterates are close to an optimal solution. Usually, fast local convergence cannot be expected if only the gradient information is used. In the Euclidean space $\mathbb{R}^{n}$, it is well known that higher rates of convergence can be achieved by using second-order information on the cost function. The classical choice is Newton's method; it plays a central role in the development of numerical techniques for optimization because of its simple formulation and its quadratic convergence properties. The history of Newton's method on manifolds can be traced back to Gabay [19] who proposed a formulation for the method on embedded submanifolds of $\mathbb{R}^{n}$. Edelman et al. [20] proposed a formulation of Newton's method on Stiefel manifold. Recently, Aihara and Sato [21] presented a matrix-free implementation of Riemannian Newton's method on the Stiefel manifold. Sato [22] developed the Riemannian Newton's method for the joint diagonalization problem on the Stiefel manifold, where the dimension of Newton's equation is reduced and can be effectively solved by means of the Kronecker product and the vec and veck operators. Very recently, $\mathrm{Hu}$ et al. [23] proposed a regularized Newton method for optimization problems on Riemannian manifolds. They used a secondorder approximation of the objective function in the Euclidean space to form a sequence of quadratic subproblems while keeping the manifold constraints. $\mathrm{Nu}-$ merical experiments and comparisons with other state-ofthe-art methods indicated that their proposed algorithm is very promising. Another popular second-order algorithm on Stiefel manifold is the Riemannian trust-region (RTR) algorithm [24-28], which has been successfully applied to various applications. In particular, Yang et al. [29] presented the RTR algorithm for $H_{2}$ model reduction of bilinear systems, where the $\mathrm{H}_{2}$ error norm is treated as a cost function on the Stiefel manifold. Sato [30] developed the RTR algorithm to the join singular value decomposition of multiple rectangular matrices, which is formulated as a Riemannian optimization problem on the product of two Stiefel manifolds.

Motivated by these works, in this paper, we are interested in extending Riemannian Newton's method and the Riemannian trust-region method to the underlying matrix trace minimization problem. We first derive the specific expression of Riemannian gradient and Hessian of the objective function of Problem 1. Specifically, we use the Kronecker product and the vectorization operators to reduce the dimension of the involved Newton's equation and to transform the equation into a standard symmetric linear system $H x=b$. The resultant system can be efficiently solved by means of direct inversion or some well-known Krylov subspace methods, such as the conjugate residual method (page 182 of [31]). Because Newton's method is not guaranteed to have global convergence, we need to prepare a suitable starting point that is sufficiently close to an optimal solution. Here, we applied OptStiefelGBB, a stateof-the-art algorithm proposed by Wen and Yin [13] which has the benefit of requiring very little memory and has been proved to be globally convergent, to obtain a suitable initial point. The resulting hybrid Riemannian Newton-type algorithm is globally and quadratically convergent. In the Euclidean space $\mathbb{R}^{n}$, it is well known that the pure Newton method converges only locally, and it cannot distinguish between local minima, local maxima, and saddle points. Compared to the pure Newton-type algorithm, the advantage of the trust-region algorithm is its more stable behavior [25]. For the RTR algorithm, convergence to stationary points is guaranteed for all initial points. By 
utilizing the Taylor expansion on Stiefel manifold, RTR algorithm constructs a trust-region subproblem on the tangent space. Here, we proposed a new trust-region subproblem, which has a lower computational cost than the original subproblem, based on our expression of the Riemannian Hessian of the objective function. The new trustregion subproblem can be solved by the classical truncated conjugate gradient, which is most popular due to its good properties and relatively cheap computational cost.

This paper is organized as follows. In Section 2, some basic geometric properties of the Stiefel manifold are given, and the representation matrix formula of Riemannian gradient and Hessian of the objective function are also derived. A hybrid Newton-type algorithm with globally and quadratically convergent is provided in Section 3. A Riemannian trustregion-based algorithm for Problem 1 is described in Section 4. Numerical experiments and application to the least squares fitting of the DEDICOM model and the orthonormal INDSCAL model and comparisons with some latest Riemannian gradient-type algorithms mentioned above and some existing second-order Riemannian algorithms in the MATALB toolbox Manopt are reported in Section 5. The conclusion is presented in Section 6.

\section{Preliminaries}

In this section, we first recall some notations, definitions, and basic properties of Riemannian manifolds used throughout the paper. The tangent space at $x$ on a manifold $\mathscr{M}$ is denoted by $\mathbf{T}_{x} \mathscr{M}$. For manifolds $\mathscr{M}$ and $\mathcal{N}$ and a mapping $f: \mathscr{M} \longrightarrow \mathscr{N}$, the differential of $f$ at $x \in \mathscr{M}$ is denoted by $\mathscr{D} f(x)$, which is a mapping from $\mathbf{T}_{x} \mathscr{M}$ to $\mathbf{T}_{f(x)} \mathscr{M}$. Given a smooth function $f$ on a manifold $\mathscr{M} \subset \mathbb{R}^{n_{1} \times n_{2}}$, the symbol $\bar{f}$ is the extension of $f$ to the ambient Euclidean space $\mathbb{R}^{n_{1} \times n_{2}}$. The symbols $\nabla$ and grad denote the Euclidean and Riemannian gradients, respectively; i.e., given a smooth function $f$ on a manifold $\mathscr{M} \subset \mathbb{R}^{n_{1} \times n_{2}}, \nabla$ and grad act on $\bar{f}$ and $f$, respectively. The symbol Hess denotes the Riemannian Hessian. The concept of a retraction, which is a smooth map from the tangent bundle of $\mathscr{M}$ into $\mathscr{M}$ that approximates the exponential map to the first order, is given as follows.

Definition 1. (see [24], Definition 4.1.1). A retraction on a differentiable manifold $\mathscr{M}$ is a smooth mapping $\mathscr{R}$ from the tangent bundle T $\mathscr{M}$ onto $\mathscr{M}$ satisfying the following two conditions (here $\mathscr{R}_{x}$ denotes the restriction of $\mathscr{R}$ to $\left.\mathrm{T}_{x} \mathscr{M}\right)$ :

(1) $\mathscr{R}_{x}\left(0_{x}\right)=x$, where $0_{x}$ denotes the zero element of $\mathrm{T}_{x} \mathscr{M}$

(2) For any $X \in \mathscr{M}$, it holds that

$$
\lim _{T_{x}, \mathscr{M} \in \xi \longrightarrow 0} \frac{\left\|\mathscr{R}_{x}(\xi)-(x+\xi)\right\|}{\|\xi\|}=0 .
$$

Given a retraction $\mathscr{R}$ and a smooth manifold $\mathscr{M}$, the general feasible algorithm framework on the manifold can be expressed as

$$
x_{k+1}=\mathscr{R}_{x}\left(t_{k} \xi_{k}\right),
$$

where $t_{k}$ is the step size at the $k$-th iterate $x_{k}$ and $\xi_{k} \in \mathbf{T}_{x_{k}} \mathscr{M}$ is a tangent vector.

We next introduce some basic geometric properties of the involved Stiefel manifold St $(n, p)$, the reader is referred to $[20,24]$ for more details. The tangent space $\mathbf{T}_{X} \operatorname{St}(n, p)$ at $X \in \operatorname{St}(n, p)$ can be expressed as

$$
\mathbf{T}_{X} \operatorname{St}(n, p)=\left\{\xi \in \mathbb{R}^{n \times p} \mid \xi^{T} X+X^{T} \xi=0\right\} .
$$

Since the manifold St $(n, p)$ is an embedded submanifold of the matrix Euclidean space $\mathbb{R}^{n \times p}$, a natural metric for $\mathbf{T}_{X} \operatorname{St}(n, p)$ is the Euclidean metric $\langle Y, Z\rangle_{X}=\operatorname{tr}\left(Y^{T} Z\right)$, which induces a norm $\|Z\|_{X}=\|Z\|_{F}$, where $\|\cdot\|_{F}$ is the Frobenius norm of a matrix. In what follows, we denote by $\langle\cdot, \cdot\rangle$ and $\|\cdot\|_{F}$ the Riemannian metric and its induced norm on $\operatorname{St}(n, p)$, respectively. Under the Riemannian metric, the orthogonal projection $\mathbf{P}_{X}$ at $X \in \operatorname{St}(n, p)$ onto $\mathbf{T}_{X} \operatorname{St}(n, p)$ is expressed as

$$
\mathbf{P}_{X}(V)=V-X \operatorname{sym}\left(X^{T} V\right), \quad \forall V \in \mathbb{R}^{n \times p},
$$

where $\operatorname{sym}(M)$ denotes the symmetric part of $M$, i.e., $\operatorname{sym}(M):=\left(\left(M+M^{T}\right) / 2\right)$. We next introduce several different retraction operators on the Stiefel manifold at the current point $X$ for a given step size $t$ and descent direction $\xi$ (one can refer to [27] for more details).

(1) Exponential map [20]:

$\mathscr{R}_{X}^{\exp }(t \xi)=[X, Q] \exp \left(t\left[\begin{array}{cc}-X^{T} \xi & -R^{T} \\ \mathbb{R} & 0\end{array}\right]\right)\left[\begin{array}{c}I_{p} \\ 0\end{array}\right]$,

where $\mathrm{QR}=-\left(I_{n}-X X^{T}\right) \xi$ is the $\mathrm{QR}$ decomposition of $-\left(I_{n}-X X^{T}\right) \xi$. This scheme needs to calculate an exponent of a $2 p$-by- $2 p$ matrix and an $\mathrm{QR}$ decomposition of an $n$-by- $p$ matrix.

(2) Cayley transformation [13]:

$\mathscr{R}_{X}^{\text {cayley }}(t \xi)=X+t P_{\xi}\left(I_{2 p}-\frac{t}{2} Q_{\xi}^{T} P_{\xi}\right)^{-1} Q_{\xi}^{T} X$,

where $P_{\xi}=\left[\Pi_{X} \xi, X\right] \in \mathbb{R}^{n \times 2 p}$ and $Q_{\xi}=\left[X,-\Pi_{X} \xi\right] \epsilon$ $\mathbb{R}^{n \times 2 p}$ with $\Pi_{X}=I_{n}-(1 / 2) X X^{T}$. When $p<(n / 2)$, this scheme is much cheaper than the exponential map.

(3) Polar decomposition (see [24], p. 58):

$$
\mathscr{R}_{X}^{\text {polar }}(t \xi)=(X+t \xi)\left(I_{p}+t^{2} \xi^{T} \xi\right)^{-(1 / 2)} .
$$

The computational cost is lower than the Cayley transform but the Cayley transformation gives a better approximation to the exponential map.

(4) QR decomposition (see [24], p. 59):

$$
\mathscr{R}_{X}^{\mathrm{QR}}(t \xi)=q f(X+t \xi),
$$

where $q f(\cdot)$ denotes the $Q$ factor of the QR decomposition of the matrix in parentheses. It can be seen as an approximation of the polar decomposition. The main cost is the $\mathrm{QR}$ decomposition of a $n$-by- $p$ matrix. 
The Riemannian gradient and Hessian of an objective function are basic concepts in Riemannian optimization; we next derive the representation matrix formulas of Riemannian gradient grad $f$ and Riemannian Hessian Hess $f$ of the objective function in Problem 1. The Riemannian gradient, grad $f(X)$, of an objective function $f$ at $X \in \operatorname{St}(n, p)$ is defined to be a unique tangent vector which satisfies

$$
\langle\operatorname{grad} f(X), \xi\rangle=\mathscr{D} f(X)[\xi], \quad \xi \in \mathbf{T}_{X} \operatorname{St}(n, p) .
$$

The Hessian, Hess $f(X)$, of $f$ at $X$ is defined to be a linear transformation of the tangent space $\mathbf{T}_{X} \mathrm{St}(n, p)$ through the covariant derivative $\nabla_{\xi}$ grad $f$ of grad $f$ evaluated at $X$ :

$$
\text { Hess } f(X)[\xi]:=\nabla_{\xi} \operatorname{grad} f, \quad \xi \in \mathbf{T}_{X} \operatorname{St}(n, p),
$$

where the covariant derivative is defined through the LeviCivita connection $\nabla$ on $\operatorname{St}(n, p)$ (see [24], Definition 5.5.1).

In what follows, we define $\bar{f}(X)$ to be a function with the same form as $f(X)$ defined in $\mathbb{R}^{n \times p}$; that is,

$$
\bar{f}(X)=c+\operatorname{tr}(A X)+\sum_{j=1}^{m} \operatorname{tr}\left(B_{j} X C_{j} X^{T}\right), \quad X \in \mathbb{R}^{n \times p} .
$$

The following two lemmas derive the explicit expressions of $\operatorname{grad} f$ and Hess $f$.
Lemma 1. The Riemannian gradient of $f$ at $X \in S t(n, p)$ can be expressed as

$$
\operatorname{grad} f(X)=\mathbf{P}_{X}\left(A^{T}+\sum_{j=1}^{m}\left(B_{j}^{T} X C_{j}^{T}+B_{j} X C_{j}\right)\right)
$$

Proof. Since St $(n, p)$ is a Riemannian submanifold of $\mathbb{R}^{n \times p}$ endowed with the induced metric, grad $f(X)$ is equal to the orthogonal projection of the Euclidean gradient $\nabla \bar{f}$ at $X$ onto $\mathbf{T}_{X} \operatorname{St}(n, p)$. On the other hand, according to the matrix trace function differentiation, the Euclidean gradient $\nabla \bar{f}$ at $X$ can be computed as

$$
\nabla \bar{f}(X)=A^{T}+\sum_{j=1}^{m}\left(B_{j}^{T} X C_{j}^{T}+B_{j} X C_{j}\right)
$$

Hence, by using the projection $\mathbf{P}_{X}$ given in (15), we obtain (23).

Lemma 2. Let $\xi$ be a tangent vector at $X \in S t(n, p)$, the Riemannian Hessian of $f$ at $X$ is expressed as a linear map on $\mathbf{T}_{X} S t(n, p)$ and given by

$$
\text { Hess } f(X)[\xi]=\mathbf{P}_{X}\left(\sum_{j=1}^{m}\left(B_{j}^{T} \xi C_{j}^{T}+B_{j} \xi C_{j}\right)-\xi \operatorname{sym}\left(X^{T} A^{T}+X^{T} \sum_{j=1}^{m}\left(B_{j}^{T} X C_{j}^{T}+B_{j} X C_{j}\right)\right)\right) \text {. }
$$

Proof. For $X \in \mathbb{R}^{n \times p}$, by simple calculation, the Euclidean Hessian $\nabla^{2} \bar{f}$ can be written as

$$
\nabla^{2} \bar{f}(X)[\xi]=\sum_{j=1}^{m}\left(B_{j}^{T} \xi C_{j}^{T}+B_{j} \xi C_{j}\right)
$$

By using the classical expression of the Riemannian connection on a Riemannian submanifold of a Euclidean space (see [32], §5.3.3) and choosing $\nabla_{\xi} \zeta:=\mathbf{P}_{X}(\mathscr{D} \zeta(X)[\xi])$, the Riemannian Hessian hess $f$ of $f$ on $\operatorname{St}(n, p)$ can also be expressed by using $\nabla^{2} \bar{f}$ and the projection map (15). That is,

$$
\text { Hess } f(X)[\xi]=\mathbf{P}_{X}(\mathscr{D}(\operatorname{grad} f)(X)[\xi]) \text {. }
$$

Note that grad $f(X)=\mathbf{P}_{X}(\nabla \bar{f}(X))$. We view the righthand side of this relation as a product of two matrix functions of $X$, rather than a composition of a map and a function. Then, (27) can be written as [32]

$$
\text { Hess } \begin{aligned}
f(X)[\xi] & =\mathbf{P}_{X}(\mathscr{D}(\operatorname{grad} f)(X)[\xi]) \\
& =\mathbf{P}_{X}\left(\mathscr{D}\left(\mathbf{P}_{X}(\nabla \bar{f})\right)(X)[\xi]\right) \\
& =\mathbf{P}_{X}\left(\mathscr{D} \mathbf{P}_{X}[\xi](\nabla \bar{f}(X))+\mathbf{P}_{X}(\mathscr{D}(\bar{f})(X)[\xi])\right) \\
& =\mathbf{P}_{X}\left(\mathscr{D} \mathbf{P}_{X}[\xi](\nabla \bar{f}(X))+\nabla^{2} \bar{f}(X)[\xi]\right),
\end{aligned}
$$

in which we used the relation $\mathbf{P}_{X} \mathbf{P}_{X}=\mathbf{P}_{X}$ in the last equality of (28), and for any $M \in \mathbb{R}^{n \times p}$,

$$
\begin{aligned}
\mathscr{D} \mathbf{P}_{X}[\xi](M) & =\mathscr{D}\left(M-X \operatorname{sym}\left(X^{T} M\right)\right)[\xi] \\
& =-\xi \operatorname{sym}\left(X^{T} M\right)-X \operatorname{sym}\left(\xi^{T} M\right) .
\end{aligned}
$$

Furthermore, if $S$ is $p \times p$ symmetric matrix, we have

$$
\mathbf{P}_{X}(X S)=X S-X \operatorname{sym}\left(X^{T} X S\right)=X S-X S=0 .
$$

This relation can reduce the computational cost of $\mathbf{P}_{X}\left(\mathscr{D} \mathbf{P}_{X}[\xi](\nabla \bar{f}(X))\right)$ in the last equality of (28). Indeed, it follows from (29) and (30) that

$$
\mathbf{P}_{X}\left(\mathscr{D} \mathbf{P}_{X}[\xi](\nabla \bar{f}(X))\right)=-\mathbf{P}_{X}\left(\xi \operatorname{sym}\left(X^{T} \nabla \bar{f}(X)\right)\right) .
$$

Consequently, by using (29) and (31), we obtain a more concrete expression for Hess $f(X)[\xi]$ acting on $\xi$ as

$$
\text { Hess } f(X)[\xi]=\mathbf{P}_{X}\left(\nabla^{2} \bar{f}(X)[\xi]-\xi \operatorname{sym}\left(X^{T} \nabla \bar{f}(X)\right)\right) \text {. }
$$

By substituting (24) and (26) into (32), we obtain (25).

At the end of this section, we introduce the vec and veck operators, which are useful for rewriting a matrix equation by transforming the matrix into an unknown column vector, 
and some useful properties will be used in the sequel. For any $M=\left(a_{i j}\right) \in \mathbb{R}^{m \times n}$, the vec operator is defined as

$$
\operatorname{vec}(M)=\left(a_{11}, \ldots, a_{m 1}, a_{12}, \ldots, a_{m 2}, a_{1 n}, \ldots, a_{m n}\right)^{T} \in \mathbb{R}^{m n} \text {. }
$$

For any $N=\left(b_{i j}\right) \in \mathbb{A} \mathbb{S}^{n \times n}$, where $\mathbb{A} \mathbb{S}^{n \times n}$ means the set of all $n$-by- $n$ skew-symmetric matrices, the veck operator is defined as

$$
\begin{aligned}
\operatorname{veck}(N)= & \sqrt{2}\left(b_{21}, b_{31}, \ldots, b_{n 1}, b_{32}, b_{42}, \ldots, b_{n 2}, \ldots,\right. \\
& \left.\cdot b_{(n-1)(n-2)}, b_{n(n-2)}, b_{n(n-1)}\right)^{T} \in \mathbb{R}^{(n(n-1) / 2)} .
\end{aligned}
$$

The above definitions yield the following inner product equivalence:

$$
\begin{aligned}
& \operatorname{tr}\left(M^{T} M^{\prime}\right)=\operatorname{vec}(M)^{T} \operatorname{vec}\left(M^{\prime}\right), \quad \forall M, M^{\prime} \in \mathbb{R}^{n \times n}, \\
& \operatorname{tr}\left(N^{T} N^{\prime}\right)=\operatorname{veck}(N)^{T} \operatorname{veck}(N), \quad \forall N, N^{\prime} \in \mathbb{A} \mathbb{S}^{n \times n} .
\end{aligned}
$$

(i) For $M \in \mathbb{R}^{m \times n}, X \in \mathbb{R}^{n \times s}$, and $M^{\prime} \in \mathbb{R}^{s \times t}$, one can get $\operatorname{vec}\left(M X M^{\prime}\right)=\left(M^{\prime T} \otimes M\right) \operatorname{vec}(X)$, where $\otimes$ is the Kronecker product. (ii) There exists an $m n \times m n$ vec-permutation matrix [33] $T_{(m, n)}=\sum_{i=1}^{n} \sum_{j=1}^{m} E_{i j} \otimes E_{i j}^{T}$ such that

$$
\operatorname{vec}(M)=T_{(m, n)} \operatorname{vec}\left(M^{T}\right), \quad M \in \mathbb{R}^{m \times n},
$$

where $E_{i j}$ is the $n \times m$ matrix with a 1 in position $(i, j)$ and zeros elsewhere. From [33], we have $T_{(m, n)} T_{(n, m)}=I_{m n}$. Because $T_{(m, n)}$ is a permutation matrix and so is orthogonal, then we have $T_{(m, n)}^{-1}=T_{(m, n)}^{T}=T_{(n, m)}$, and then

$$
\operatorname{vec}\left(M^{T}\right)=T_{(n, m)} \operatorname{vec}(M) .
$$

(iii) For $M \in \mathbb{R}^{n \times n}$, let skew $(M)$ be the skew-symmetric parts of $M$, i.e., skew $(M):=\left(\left(M-M^{T}\right) / 2\right)$, and we have

$$
\operatorname{vec}(\operatorname{skew}(M))=\frac{1}{2}\left(I_{n^{2}}-T_{(n, n)}\right) \operatorname{vec}(M)
$$

(iv) For $N \in \mathbb{A} \mathbb{S}^{n \times n}$, we have

$$
\operatorname{vec}(N)=K_{n} \operatorname{veck}(N),
$$

where the matrix $K_{n} \in \mathbb{R}^{n^{2} \times(n(n-1) / 2)}$ is defined as the following form:

$$
K_{n}=\frac{1}{\sqrt{2}}\left(\begin{array}{ccccccccccc}
e_{2} & e_{3} & \cdots & e_{n-1} & e_{n} & 0 & \cdots & 0 & 0 & \cdots & 0 \\
-e_{1} & 0 & \cdots & 0 & 0 & e_{3} & \cdots & e_{n-1} & e_{n} & \cdots & 0 \\
0 & -e_{1} & \cdots & 0 & 0 & -e_{2} & \cdots & 0 & 0 & \cdots & 0 \\
\vdots & \vdots & & \vdots & \vdots & \vdots & & \vdots & \vdots & & \vdots \\
0 & 0 & \cdots & -e_{1} & 0 & 0 & \cdots & -e_{2} & 0 & \cdots & e_{n} \\
0 & 0 & \cdots & 0 & -e_{1} & 0 & \cdots & 0 & -e_{2} & \cdots & -e_{n-1}
\end{array}\right),
$$

where $e_{i}$ is the $i$ th column of $I_{n}$. Obviously, $K_{n}$ is standard column orthogonal, that is, $K_{n}^{T} K_{n}=$ $I_{(n(n-1) / 2)}$. Furthermore, for $N \in \mathbb{A} \mathbb{S}^{n \times n}$, we have

$$
\operatorname{veck}(N)=K_{n}^{T} \operatorname{vec}(N) \text {. }
$$

\section{Riemannian Newton's Method for Problem 1}

Since we have already obtained the matrix expressions of grad $f$ and Hess $f$ and some other requisites for Riemannian optimization algorithms, in this section, we develop Riemannian Newton's method for Problem 1. In Newton's method, the search direction $\xi^{(k)} \in \mathbf{T}_{X^{(k)}} \operatorname{St}(n, p)$ at $X^{(k)} \in$ $\operatorname{St}(n, p)$ is determined to be the solution to Newton's equation

$$
\text { Hess } f\left(X^{(k)}\right)[(\xi)]=-\operatorname{grad} f\left(X^{(k)}\right) .
$$

After $\xi^{(k)}$ is obtained, the candidate for the new iterate is then given by where the step size is set to one for simplicity. By substituting (23) and (25) into (43), complete Riemannian Newton's equation for Problem 1 at $X \in \operatorname{St}(n, p)$ can be written as

$$
\begin{aligned}
\mathbf{P}_{X}\left(\sum_{j=1}^{m}\left(B_{j}^{T} \xi C_{j}^{T}+B_{j} \xi C_{j}\right)\right. \\
\left.\quad-\xi \operatorname{sym}\left(X^{T} A^{T}+X^{T} \sum_{j=1}^{m}\left(B_{j}^{T} X C_{j}^{T}+B_{j} X C_{j}\right)\right)\right) \\
=-\mathbf{P}_{X}\left(A^{T}+\sum_{j=1}^{m}\left(B_{j}^{T} X C_{j}^{T}+B_{j} X C_{j}\right)\right) .
\end{aligned}
$$

Now, the problem is how to solve the above Newton's equation. Noting that it is complicated and difficult to solve because it must be solved for $\xi \in \mathbf{T}_{X} \operatorname{St}(n, p)$ for given 
$X \in \operatorname{St}(n, p)$, i.e., $\xi$ must satisfy $\xi^{T} X+X^{T} \xi=0$. To overcome these difficulties, we wish to obtain the representation matrix of Hess $f$ as a linear transformation on $\mathbf{T}_{X} \operatorname{St}(n, p)$ for arbitrarily fixed $X$ so that we can rewrite (45) into a standard linear equation, which permits many quite effective numerical approaches. To this end, we introduce the following lemma.

Lemma 3 (see [24], p. 42). An equivalent form of the tangent space to $\operatorname{St}(n, p)$ at $X \in S t(n, p)$ is given by

$$
\mathbf{T}_{X} \operatorname{St}(n, p)=\left\{X U+X_{\perp} V \mid U \in \mathbb{A} \mathbb{S}^{p \times p}, \quad V \in \mathbb{R}^{(n-p) \times p}\right\},
$$

where $X_{\perp}$ is an arbitrary $n \times(n-p)$ matrix that satisfies $X^{T} X_{\perp}=0$ and $X_{\perp}^{T} X_{\perp}=I_{n-p}$.

From Lemma 3, one can easily check that the vector space of all tangent vectors has a dimension of $n p-(1 / 2) p(p+1)$. Let $\xi \in \mathbf{T}_{X} \operatorname{St}(n, p)$ be the search direction at $X \in \operatorname{St}(n, p)$. From Lemma $3, \xi$ can be expressed by

$$
\xi=X U+X_{\perp} V, \quad U \in \mathbb{A} \mathbb{S}^{p \times p}, V \in \mathbb{R}^{(n-p) \times p} .
$$

Since Hess $f(X)[\xi]$ is also determined to be a unique tangent vector in $\mathbf{T}_{X} \mathrm{St}(n, p)$, there exist unique matrices $U_{H} \in \mathbb{A} \mathbb{S}^{p \times p}$ and $V_{H} \in \mathbb{R}^{(n-p) \times p}$ which satisfy

$$
\text { Hess } f(X)[\xi]=X U_{H}+X_{\perp} V_{H} \text {. }
$$

The following proposition shows that we can write $U_{H}$ and $V_{H}$ by using $U$ and $V$.

Proposition 1. Let $X \in S t(n, p)$ and $X_{\perp} \in S t(n-p, p)$ satisfy $X^{T} X_{\perp}=0$. If a tangent vector $\xi \in \mathbf{T}_{X} S t(n, p)$ is expressed as (47), then the Hessian Hess $f(X)$ of the objective function acts on $\xi$ as Hess $f(X)[\xi]=X U_{H}+X_{\perp} V_{H}$ with

$$
\begin{aligned}
& \mathbf{U}_{\mathbf{H}}=\operatorname{skew}\left(\sum_{j=1}^{m}\left(X^{T} B_{j}^{T} X \mathbf{U} C_{j}^{T}+X^{T} B_{j} X \mathbf{U} C_{j}+X^{T} B_{j}^{T} X_{\perp} \mathbf{V} C_{j}^{T}+X^{T} B_{j} X_{\perp} \mathbf{V} C_{j}\right)-\mathbf{U} \operatorname{sym}\left(X^{T} A^{T}+X^{T} \sum_{j=1}^{m}\left(B_{j}^{T} X C_{j}^{T}+B_{j} X C_{j}\right)\right),\right. \\
& \mathbf{V}_{\mathbf{H}}=\sum_{j=1}^{m}\left(X_{\perp}^{T} B_{j}^{T} X \mathbf{U} C_{j}^{T}+X_{\perp}^{T} B_{j} X \mathbf{U} C_{j}+X_{\perp}^{T} B_{j}^{T} X_{\perp} \mathbf{V} C_{j}^{T}+X_{\perp}^{T} B_{j} X_{\perp} \mathbf{V} C_{j}\right)-\mathbf{V} \operatorname{sym}\left(X^{T} A^{T}+X^{T} \sum_{j=1}^{m}\left(B_{j}^{T} X C_{j}^{T}+B_{j} X C_{j}\right)\right) .
\end{aligned}
$$

Proof. From (25) and (48), we have the following equality:

$$
X U_{H}+X_{\perp} V_{H}=\mathbf{P}_{X}\left(\sum_{j=1}^{m}\left(B_{j}^{T} \xi C_{j}^{T}+B_{j} \xi C_{j}\right)-\xi \operatorname{sym}\left(X^{T} A^{T}+X^{T} \sum_{j=1}^{m}\left(B_{j}^{T} X C_{j}^{T}+B_{j} X C_{j}\right)\right)\right)
$$

Note that $X^{T} \mathbf{P}_{X}(W)=\operatorname{skew}\left(X^{T} W\right)$ and $X_{\perp}^{T} \mathbf{P}_{X}(W)=$ $X_{\perp}^{T} W$ for any $W \in \mathbb{R}^{n \times p}$. Multiplying equation (51) by $X^{T}$ from the left and using the relations $X^{T} X=I_{p}$ and $X^{T} X_{\perp}=$ 0 yields

$$
\begin{aligned}
U_{H} & =X^{T} \mathbf{P}_{X}\left(\sum_{j=1}^{m}\left(B_{j}^{T} \xi C_{j}^{T}+B_{j} \xi C_{j}\right)-\xi \operatorname{sym}\left(X^{T} A^{T}+X^{T} \sum_{j=1}^{m}\left(B_{j}^{T} X C_{j}^{T}+B_{j} X C_{j}\right)\right)\right) \\
& =\operatorname{skew}\left(X^{T} \sum_{j=1}^{m}\left(B_{j}^{T} \xi C_{j}^{T}+B_{j} \xi C_{j}\right)-X^{T} \xi \operatorname{sym}\left(X^{T} A^{T}+X^{T} \sum_{j=1}^{m}\left(B_{j}^{T} X C_{j}^{T}+B_{j} X C_{j}\right)\right)\right) .
\end{aligned}
$$

By substituting (47) into (52) and using the relation $X^{T} \xi=X^{T}\left(X U+X_{\perp} V\right)=U$, we obtain the expression of $U_{H}$ in (49) by using $U$ and $V$. Similarly, we multiply (51) by $X_{\perp}^{T}$ from the left to obtain 


$$
\begin{array}{r}
V_{H}=X_{\perp}^{T} \mathbf{P}_{X}\left(\sum_{j=1}^{m}\left(B_{j}^{T} \xi C_{j}^{T}+B_{j} \xi C_{j}\right)-\xi \operatorname{sym}\left(X^{T} A^{T}+X^{T} \sum_{j=1}^{m}\left(B_{j}^{T} X C_{j}^{T}+B_{j} X C_{j}\right)\right)\right) \\
=X_{\perp}^{T} \sum_{j=1}^{m}\left(B_{j}^{T} \xi C_{j}^{T}+B_{j} \xi C_{j}\right)-X_{\perp}^{T} \xi \operatorname{sym}\left(X^{T} A^{T}+X^{T} \sum_{j=1}^{m}\left(B_{j}^{T} X C_{j}^{T}+B_{j} X C_{j}\right)\right) .
\end{array}
$$

By substituting (47) into (53) and using the relation $X_{\perp}^{T} \xi=X_{\perp}^{T}\left(X U+X_{\perp} V\right)=V$, we obtain the expression of $V_{H}$ in $(50)$.

Since the Hessian Hess $f(X)[\xi]$ is a linear transformation with respect to $\xi=X U+X, V$, and, therefore, with respect to the set of matrices $(U, V)$ with $U \in A \mathbb{S}^{p \times p}$ and $V \in \mathbb{R}^{n-p \times p}$. Meanwhile, Hess $f(X)[\xi]$ is also in the tangent space $\mathbf{T}_{X} \mathrm{St}(n, p)$ and can be uniquely expressed as Hess $f(X)[\xi]=X U_{H}+X_{\perp} V_{H}$ with $U_{H} \in \mathbb{A} \mathbb{S}^{p \times p}, V_{H} \in$ $\mathbb{R}^{(n-p) \times p}$. Note that the dimension of the tangent space $\mathbf{T}_{X} \operatorname{St}(n, p)$ is $K:=(p(p-1) / 2)+p(n-p)$; then, we can regard that the Hessian Hess $f(X)[\xi]$ is a linear transformation on $\mathbb{R}^{K}$ that transforms a $K$-dimension vector into another $K$-dimension vector. The following lemma obtains the representation matrix of that linear transformation, in which we use the vec and veck operators taking matrices and skew-symmetric matrices into vectors.

Lemma 4. Let $H$ be a linear transformation on $\mathbb{R}^{K}$ that acts on $\left(\operatorname{veck}(U)^{T}, \operatorname{vec}(V)^{T}\right)^{T}$ with $U \in \mathbb{A} \mathbb{S}^{p \times p}, V \in \mathbb{R}^{p(n-p)}$ as

$$
H\left(\begin{array}{c}
\operatorname{veck}(U) \\
\operatorname{vec}(V)
\end{array}\right)=\left(\begin{array}{c}
\operatorname{veck}\left(U_{H}\right) \\
\operatorname{vec}\left(V_{H}\right)
\end{array}\right),
$$

where $U_{H}$ and $V_{H}$ are given in equations (49) and (50). Then, the representation matrix $H_{A}$ of $H$ is given by

$$
H_{A}=\left(\begin{array}{ll}
H_{11} & H_{12} \\
H_{21} & H_{22}
\end{array}\right) \text {, }
$$

where

$$
\begin{aligned}
H_{11}= & \frac{1}{2} K_{p}^{T}\left(I_{p^{2}}-T_{(p, p)}\right)\left(\sum_{j=1}^{m}\left(C_{j} \otimes\left(X^{T} B_{j}^{T} X\right)+C_{j}^{T} \otimes\left(X^{T} B_{j} X\right)\right)-\operatorname{sym}\left(X^{T} A^{T}+X^{T} \sum_{j=1}^{m}\left(B_{j}^{T} X C_{j}^{T}+B_{j} X C_{j}\right)\right) \otimes I_{p}\right) K_{p}, \\
H_{12}= & \frac{1}{2} K_{p}^{T}\left(I_{p^{2}}-T_{(p, p)}\right)\left(\sum_{j=1}^{m}\left(C_{j} \otimes\left(X^{T} B_{j}^{T} X_{\perp}\right)+C_{j}^{T} \otimes\left(X^{T} B_{j} X_{\perp}\right)\right)\right) \\
H_{21}= & \sum_{j=1}^{m}\left(C_{j} \otimes\left(X_{\perp}^{T} B_{j}^{T} X\right)+C_{j}^{T} \otimes\left(X_{\perp}^{T} B_{j} X\right)\right) K_{p}, \\
H_{22}= & \sum_{j=1}^{m}\left(C_{j} \otimes\left(X_{\perp}^{T} B_{j}^{T} X_{\perp}\right)+C_{j}^{T} \otimes\left(X_{\perp}^{T} B_{j} X_{\perp}\right)\right)-\operatorname{sym}\left(X^{T} A^{T}+X^{T} \sum_{j=1}^{m}\left(B_{j}^{T} X C_{j}^{T}+B_{j} X C_{j}\right)\right) \otimes I_{n-p} .
\end{aligned}
$$

Proof. From equations (49) and (50) together with (39)-(42), $\operatorname{veck}\left(U_{H}\right)$ and $\operatorname{vec}\left(V_{H}\right)$ are calculated as follows:

$$
\begin{aligned}
\operatorname{veck}\left(U_{H}\right)= & K_{p}^{T} \operatorname{vec}\left(U_{H}\right) \\
= & K_{p}^{T} \operatorname{vec}\left(\operatorname { s k e w } \left(\sum_{j=1}^{m}\left(X^{T} B_{j}^{T} X U C_{j}^{T}+X^{T} B_{j} X U C_{j}+X^{T} B_{j}^{T} X_{\perp} V C_{j}^{T}+X^{T} B_{j} X_{\perp} V C_{j}\right)\right.\right. \\
& \left.\left.-U \operatorname{sym}\left(X^{T} A^{T}+X^{T} \sum_{j=1}^{m}\left(B_{j}^{T} X C_{j}^{T}+B_{j} X C_{j}\right)\right)\right)\right) \\
& =\frac{1}{2} K_{p}^{T}\left(I_{p^{2}}-T_{(p, p)}\right)\left(\sum_{j=1}^{m}\left(C_{j} \otimes\left(X^{T} B_{j}^{T} X\right)+C_{j}^{T} \otimes\left(X^{T} B_{j} X\right)\right) \operatorname{vec}(U)+\left(C_{j} \otimes\left(X^{T} B_{j}^{T} X_{\perp}\right)+C_{j}^{T} \otimes\left(X^{T} B_{j} X_{\perp}\right)\right) \operatorname{vec}(V)\right.
\end{aligned}
$$




$$
\begin{aligned}
& \left.-\left(\operatorname{sym}\left(X^{T} A^{T}+X^{T} \sum_{j=1}^{m}\left(B_{j}^{T} X C_{j}^{T}+B_{j} X C_{j}\right)\right) \otimes I_{p}\right) \operatorname{vec}(U)\right) \\
= & H_{11} \operatorname{veck}(U)+H_{12} \operatorname{vec}(V), \\
\operatorname{vec}\left(V_{H}\right)= & \operatorname{vec}\left(\sum_{j=1}^{m}\left(X_{\perp}^{T} B_{j}^{T} X U C_{j}^{T}+X_{\perp}^{T} B_{j} X U C_{j}+X_{\perp}^{T} B_{j}^{T} X_{\perp} V C_{j}^{T}+X_{\perp}^{T} B_{j} X_{\perp} V C_{j}\right)\right. \\
& \left.-V \operatorname{sym}\left(X^{T} A^{T}+X^{T} \sum_{j=1}^{m}\left(B_{j}^{T} X C_{j}^{T}+B_{j} X C_{j}\right)\right)\right) \\
= & \sum_{j=1}^{m}\left(C_{j} \otimes\left(X_{\perp}^{T} B_{j}^{T} X\right)+C_{j}^{T} \otimes\left(X_{\perp}^{T} B_{j} X\right)\right) \operatorname{vec}(U) \\
& +\left(C_{j} \otimes\left(X_{\perp}^{T} B_{j}^{T} X_{\perp}\right)+C_{j}^{T} \otimes\left(X_{\perp}^{T} B_{j} X_{\perp}\right)\right) \operatorname{vec}(V)-\left(\operatorname{sym}\left(X^{T} A^{T}+X^{T} \sum_{j=1}^{m}\left(B_{j}^{T} X C_{j}^{T}+B_{j} X C_{j}\right)\right) \otimes I_{n-p}\right) \operatorname{vec}(V) \\
= & H_{21} \operatorname{veck}(U)+H_{22} \operatorname{vec}(V) .
\end{aligned}
$$

This completes the proof.

The following proposition considers the structure of the representation matrix $H_{A}$.

Proposition 2. The representation matrix $H_{A}$ is always symmetric.

Proof. Let $\xi$ and $\eta$ be two tangent vectors at $X$ which are expressed by

$$
\begin{aligned}
& \xi=X U_{1}+X_{\perp} V_{1}, \\
& \eta=X U_{2}+X_{\perp} V_{2},
\end{aligned}
$$

where $U_{1}, U_{2} \in \mathbb{A} \mathbb{S}^{p \times p}$ and $V_{1}, V_{2} \in \mathbb{R}^{(n-p) \times p}$. From the induced metric for $\mathbf{T}_{X} \mathrm{St}(n, p)$, together with (35) and (36), one can get

$$
\begin{aligned}
\langle\xi, \eta\rangle & =\operatorname{tr}\left(U_{1}^{T} U_{2}\right)+\operatorname{tr}\left(V_{1}^{T} V_{2}\right) \\
& =\operatorname{veck}\left(U_{1}\right)^{T} \operatorname{veck}\left(U_{2}\right)+\operatorname{vec}\left(V_{1}\right)^{T} \operatorname{vec}\left(V_{2}\right) .
\end{aligned}
$$

Since the Hessian Hess $f(X)$ is symmetric with respect to the induced inner product for $\mathbf{T}_{X} \mathrm{St}(n, p)$ [24], we have

$\langle\operatorname{Hess} f(X)[\xi], \eta\rangle=\langle\operatorname{Hess} f(X)[\eta], \xi\rangle, \quad \xi, \eta \in \mathbf{T}_{X} \operatorname{St}(n, p)$.

This is equivalent to

$$
\begin{aligned}
& \left(\begin{array}{c}
\operatorname{veck}\left(U_{1}\right) \\
\operatorname{vec}\left(V_{1}\right)
\end{array}\right)^{T} H_{A}\left(\begin{array}{c}
\operatorname{veck}\left(U_{2}\right) \\
\operatorname{vec}\left(V_{2}\right)
\end{array}\right) \\
& =\left(H_{A}\left(\begin{array}{c}
\operatorname{veck}\left(U_{1}\right) \\
\operatorname{vec}\left(V_{1}\right)
\end{array}\right)\right)^{T}\left(\begin{array}{c}
\operatorname{veck}\left(U_{2}\right) \\
\operatorname{vec}\left(V_{2}\right)
\end{array}\right) .
\end{aligned}
$$

Hence, we obtain

$$
H_{A}=H_{A}^{T}
$$

because $\xi$ and $\eta$ can be arbitrary tangent vectors at $X$.

We are now in the position to solve Newton's equation (45). From (48), Newton's equation (45) can be rewritten as

$$
X U_{H}+X_{\perp} V_{H}=-\mathbf{P}_{X}\left(A^{T}+\sum_{j=1}^{m}\left(B_{j}^{T} X C_{j}^{T}+B_{j} X C_{j}\right)\right) .
$$

By multiplying (63) from the left by $X^{T}$ and $X_{\perp}^{T}$, (63) is equivalent to

$$
\left\{\begin{array}{l}
U_{H}=-X^{T} \mathbf{P}_{X}\left(A^{T}+\sum_{j=1}^{m}\left(B_{j}^{T} X C_{j}^{T}+B_{j} X C_{j}\right)\right), \\
V_{H}=-X_{\perp}^{T} \mathbf{P}_{X}\left(A^{T}+\sum_{j=1}^{m}\left(B_{j}^{T} X C_{j}^{T}+B_{j} X C_{j}\right)\right) .
\end{array}\right.
$$

Note again that $X^{T} \mathbf{P}_{X}(W)=\operatorname{skew}\left(X^{T} W\right)$ and $X_{\perp}^{T} \mathbf{P}_{X}(W)=X_{\perp}^{T} W$ for any $W \in \mathbb{R}^{n \times p}$. Applying the vec operation to the two equations of (64), we get 


$$
\left\{\begin{array}{l}
\operatorname{veck}\left(U_{H}\right)=-K_{p}^{T}\left(I_{p^{2}}-T_{(p, p)}\right) \operatorname{vec}\left(X^{T} A^{T}+X^{T} \sum_{j=1}^{m}\left(B_{j}^{T} X C_{j}^{T}+B_{j} X C_{j}\right)\right), \\
\operatorname{vec}\left(V_{H}\right)=-\operatorname{vec}\left(X_{\perp}^{T} A^{T}+X_{\perp}^{T} \sum_{j=1}^{m}\left(B_{j}^{T} X C_{j}^{T}+B_{j} X C_{j}\right)\right) .
\end{array}\right.
$$

Together with Lemma 4, Newton's equation (45) can be represented in the following form:

$$
H_{A} x=g \text {, }
$$

where $H_{A}$ is given in (55), which is the representation symmetric matrix of Hess Hess $f(X)$ :

$$
\begin{aligned}
& x=\left(\begin{array}{c}
\operatorname{veck}(U) \\
\operatorname{vec}(V)
\end{array}\right), \\
& g=-\left(\begin{array}{c}
K_{p}^{T}\left(I_{p^{2}}-T_{(p, p)}\right) \operatorname{vec}\left(X^{T} A^{T}+X^{T} \sum_{j=1}^{m}\left(B_{j}^{T} X C_{j}^{T}+B_{j} X C_{j}\right)\right) \\
\operatorname{vec}\left(X_{\perp}^{T} A^{T}+X_{\perp}^{T} \sum_{j=1}^{m}\left(B_{j}^{T} X C_{j}^{T}+B_{j} X C_{j}\right)\right)
\end{array}\right) .
\end{aligned}
$$

We have thus derived an equivalent form, which is a standard symmetric linear equation, of Newton's equation (45). After we solve this symmetric linear equation (66), we can obtain veck $(U)$ and vec $(V)$; then, we can easily reshape $U \in \mathbb{A}^{\mathbb{S}^{p \times p}}$ and $V \in \mathbb{R}^{(n-p) \times p}$. Therefore, we can calculate the solution $\xi=X U+X_{\perp} V$ of Newton's equation (45).

Based on the above discussion, the corresponding Riemannian Newton's method for solving Problem 1 can be described as follows.

If we know a good approximate solution of the problem in advance, Newton's method works effectively. This is because Newton's method generates locally but quadratically convergent sequences in general (Theorem 6.3.2 of $[24,34]$ ).

Theorem 1. (local convergence). Consider Algorithm 1 with retraction $\mathscr{R}$ as described in Section 2. Let $X^{*} \in S t(n, p)$ be a critical point of $f$; grad $f\left(X^{*}\right)=0$. Assume that Hess $f\left(X^{*}\right)$ is nondegenerate at $X^{*} \in S t(n, p)$. Then, there exists a neighborhood $U$ of $X^{*}$ in $S t(n, p)$ such that for all $X^{(0)} \in U$, Algorithm 1 generates an infinite sequence $\left\{X^{(k)}\right\}$ converging quadratically to $X^{*}$.

From [17, 21, 22], we have several remarks for Algorithm 1 as follows.

Remark 1. In the Step 3 of Algorithm 1, we can arbitrarily fix $X_{\perp}^{(k)}$ to satisfy $\left(X^{(k)}\right)^{T} X_{\perp}^{(k)}=0$ and $\left(X_{\perp}^{(k)}\right)^{T} X_{\perp}^{(k)}=I_{n-p}$. Such $X_{\perp}^{(k)}$ can be computed by the full QR decompositions. In practice, using the MATLAB's qr function with input $X^{(k)} \in \operatorname{St}(n, p)$ returns an orthogonal matrix $Q \in \mathbb{R}^{n \times n}$ such that $X^{(k)}=Q\left(\begin{array}{c}R_{1} \\ 0\end{array}\right)$ where $R_{1}$ is a $p \times p$ upper triangular matrix. If we partition $Q=\left(Q_{1}, Q_{2}\right)$ with $Q_{1} \in \operatorname{St}(n, p)$ and
$Q_{2} \in \operatorname{St}(n, n-p), \quad$ then $\quad Q_{2}^{T} X^{(k)}=\left(0, I_{n-p}\right)\left(\begin{array}{c}R_{1} \\ 0\end{array}\right)=0$.

Therefore, we can choose $Q_{2}$ as $X_{\perp}^{(k)}$.

Remark 2. The symmetric linear equation (69) can be solved by direct inversion if $H_{A}^{(k)}$ is invertible. However, if the dimension of the problem is large, it is difficult to equate by direct inversion. In this case, some Krylov subspace methods such as the conjugate residual (CR) method (page 182 of [31]) can be used to solve (69). The $\mathrm{CR}$ method is used to solve linear equations of the form $A x=b$ where $A$ is an invertible and symmetric matrix and $b$ is nonzero. Note that the system matrix is only required to be symmetric, not symmetric positive definite. However, we should point out that other solvers can also be applied to solve (69) because there are no specific structural requirements for the variable $x$. When Hess $f(X)$ is positive definite, the conjugate gradient (CG) method can be used. Moreover, if we consider (69) as just a linear equation of standard form, then the representation matrix $H_{A}$ is not necessarily symmetric. In this case, more Krylov subspace methods can be used, such as the generalized minimal residual (GMRES) method and biconjugate gradient stabilized (BiCGSTAB) method. Specifically, we use the CR method for all the numerical experiments in Section 5.

Remark 3. In general, the Newton vector $\xi^{(k)}$, solution of (43), is not necessarily a descent direction of $f(X)$. Indeed, we have 
(1) Choose an initial point $X^{(0)} \in \operatorname{St}(n, p)$.

(2) for $k=0,1,2, \ldots$ do

(3) Compute $X_{\perp}^{(k)}$ that satisfies $\left(X^{(k)}\right)^{T} X_{\perp}^{(k)}=0$ and $\left(X_{\perp}^{(k)}\right)^{T} X_{\perp}^{(k)}=I_{n-p}$.

(4) Compute the partition matrix $H_{A}^{(k)}$ by Lemma 4 and the vector $g^{(k)}$ by (68). Compute $x^{(k)} \in \mathbb{R}^{n p-(1 / 2) p(p+1)}$ that satisfies the symmetric linear equation $H_{A}^{(k)} x^{(k)}=g^{(k)}(69)$.

(5) Partition $x^{(k)} \in \mathbb{R}^{n p-(1 / 2) p(p+1)}$ as $x^{(k)^{T}}=\left(x_{1}^{(k)^{T}}, x_{2}^{(k)^{T}}\right)$, where $x_{1}^{(k)} \in \mathbb{R}^{(p(p-1) / 2)}, x_{2}^{(k)} \in \mathbb{R}^{(n-p) p}$.

(6) Construct $U^{(k)} \in \mathbb{A} \mathbb{S}^{p \times p}$ and $V^{(k)} \in \mathbb{R}^{(n-p) \times p}$ that satisfy veck $\left(U^{(k)}\right)=x_{1}^{(k)}$ and $\operatorname{vec}\left(V^{(k)}\right)=x_{2}^{(k)}$.

(7) Compute $\xi^{(k)}=X^{(k)} U^{(k)}+X_{\perp}^{(k)} V^{(k)}$.

(8) Compute the next iterate $X^{(k+1)}=\mathscr{R}_{X^{(k)}}\left(\xi^{(k)}\right)$, where $\mathscr{R}$ is a retraction on $\operatorname{St}(n, p)$.

(9) end for

Algorithm 1: Riemannian Newton's method for Problem 1.

$$
\begin{aligned}
\mathscr{D} f(X)[\xi] & =\langle\operatorname{grad} f(X), \xi\rangle \\
& =-\left\langle\operatorname{grad} f(X), \text { Hess } f(X)^{-1} \operatorname{grad} f(X)\right\rangle,
\end{aligned}
$$

which is not guaranteed to be negative without additional assumptions on the operator Hess $f(X)$. A sufficient condition for $\xi^{(k)}$ to be a descent direction is that Hess $f\left(X^{(k)}\right)$ be positive definite, i.e., $\langle\xi$, Hess $f(X)[\xi]\rangle>0$ for all $\xi \neq 0$. The positive definiteness of the representation matrix $H_{A}$ can be used to check the positive definiteness of the Hessian Hess $f(X)$, which can be used to check whether a critical point obtained by Algorithm 1 is a local minimum. We note that for any $\xi=X U_{1}+X_{\perp} V_{1} \in \mathbf{T}_{X} \operatorname{St}(n, p)$ with $U_{1} \in \mathbb{A}^{p \times p}, V_{1} \in$ $\mathbb{R}^{(n-p) \times p}$,

$$
\langle\text { Hess } f(X)[\xi], \xi\rangle=\left(\begin{array}{c}
\operatorname{veck}\left(U_{1}\right) \\
\operatorname{vec}\left(V_{1}\right)
\end{array}\right)^{T} H_{A}\left(\begin{array}{c}
\operatorname{veck}\left(U_{1}\right) \\
\operatorname{vec}\left(V_{1}\right)
\end{array}\right) \text {. }
$$

Therefore, if the symmetric matrix $H_{A}$ is positive definite, then Hess $f(X)$ is positive definite, and $X$ is a local minimum. By using the partition form (55) of $H_{A}$, we can easily derive a necessary condition for the positive definiteness of $H_{A}$ as $H_{11}$ and $H_{22}$ are positive definite. We can obtain from this fact an easy way to check the necessity for the positive definiteness as all the diagonal components of $H_{11}$ and $H_{22}$ are positive. We next consider the case where Algorithm 1 arrives at a critical point of $f$ and assume that $H_{A}$ is semipositive definite. Then, $H_{A}$ is positive definite if and only if $0 \neq \operatorname{det}\left(H_{A}\right)=\operatorname{det}\left(H_{11}\right) \operatorname{det}\left(H_{22}-H_{21} H_{11}^{-1} H_{12}\right)$. Therefore, under the assumption of semipositive definiteness of $H_{A}$, the condition of positive definiteness of $H_{A}$ is necessary and sufficient for $\operatorname{det}\left(H_{11}\right) \neq 0$ and $\operatorname{det}\left(H_{22}-\right.$ $\left.H_{21} H_{11}^{-1} H_{12}\right) \neq 0$.

Remark 4. Similar to unconstrained optimization in the Euclidean space, $\left\|\operatorname{grad} f\left(X^{(k)}\right)\right\|_{F}<\varepsilon$ for some constant $\varepsilon>0$ is a reasonable stopping criterion for Problem 1. In fact, the Lagrangian of Problem 1 is

$$
\mathscr{L}(X, \Lambda)=\bar{f}(X)-\frac{1}{2} \operatorname{tr}\left(\Lambda\left(X^{T} X-I_{p}\right)\right),
$$

where $\Lambda$ is a symmetric matrix representing the Lagrange multiply. Then, the first-order optimality conditions in the Euclidean sense are

$$
\begin{aligned}
X^{T} X & =I_{p}, \\
\frac{\partial}{\partial X} \mathscr{L}(X, \Lambda) & =\nabla \bar{f}(X)-X \Lambda=0 .
\end{aligned}
$$

From the second equation, we have $\Lambda=X^{T} \nabla \bar{f}(X)$. Since $\Lambda$ must be symmetric, we further obtain $\Lambda=\nabla \bar{f}$ $(X)^{T} X$. Under the conditions $X^{T} X=I_{p},(\partial / \partial X) \mathscr{L}(X, \Lambda)=$ 0 is equivalent to $\operatorname{grad} f(X)=0$, since it follows from (15), (23), and (24) that

$$
\begin{aligned}
\mathbf{P}_{X}(\nabla \bar{f}(X)) & =\left(I_{n}-\frac{1}{2} X X^{T}\right)\left(\nabla \bar{f}(X)-X \nabla \bar{f}(X)^{T} X\right) \\
& =\left(I_{n}-\frac{1}{2} X X^{T}\right) \frac{\partial}{\partial X} \mathscr{L}(X, \Lambda) .
\end{aligned}
$$

Thus, first-order critical points in the Euclidean sense can be interpreted as stationary points in the Riemannian sense.

Because Newton's method is not guaranteed to have global convergence, we need to prepare a suitable starting point that is sufficiently close to an optimal solution. Here, we apply a state-of-the-art Riemannian gradient-type algorithm, OptStiefelGBB, to obtain a suitable initial point. OptStiefelGBB, proposed by Wen and Yin [13], is simple and has the benefit of requiring very little memory. The retraction adapted in OptStiefelGBB is the Cayley transformation (17), and an initial step size computed by the Barzilai-Borwein (BB) method [35] is used to speed up the convergence. Since the BB step size does not necessarily decrease the objective value at every iteration, it may invalidate convergence, and a nonmonotone line search method based on a strategy in [36] is also used to guarantee global convergence.

To speed up the convergence, we propose a hybrid method for Problem 1. The strategy is as follows: we start to perform the OptStiefelGBB until $\left\|\operatorname{grad} f\left(X^{(k)}\right)\right\|_{F}$ is sufficiently small. Then, we switch the method to Newton's one. One possibility of the switching criterion can be set as 


$$
\left\|\operatorname{grad} f\left(X^{(k)}\right)\right\|_{F} \leq \varepsilon_{1},
$$

where $\varepsilon_{1}>0$. The algorithm is stated as follows.

Since the cost function $f(X)$ in Problem 1 is smooth and the Stiefel manifold $\mathrm{St}(n, p)$ is compact, OptStiefelGBB applied to Problem 1 generates a sequence converging globally to a critical point of $f$ [13]. On the other hand, according to Theorem 1, Newton's method generates a quadratically convergent sequence if the initial point is sufficiently close to an optimal solution. Therefore, we know that the proposed hybrid algorithm is globally and quadratically convergent if $\varepsilon_{1}$ is sufficiently small.

Proposition 3. If $\varepsilon_{1}>0$ is sufficiently small, Algorithm 2 with the switching criterion (74) generates a globally and quadratically convergent sequence.

\section{Riemannian Trust-Region Method for Problem 1}

With the preliminary results discussed in Section 2, we now state the Riemannian trust-region method for solving Problem 1. The trust-region method is an iterative method for minimizing a cost function. At each iteration step, a quadratic model of the cost function is obtained. This model is assumed to be suitable in a region (the trust region) around the current iterate. Then, an update is computed as the minimizer of the model in the trust region. The quality of the trial update is evaluated; it is consequently accepted or rejected, and the trust-region radius is adjusted.
In a Euclidean space $E$, if $f: E \longrightarrow \mathbb{R}$ and $\langle\cdot, \cdot\rangle$ is the inner product in $E$, the trust-region subproblem for finding the update $\xi \in E$ for the current iterate $x_{k} \in E$ is given by

$$
\begin{aligned}
& \min _{\xi \in E} \quad m(\xi)=f\left(x_{k}\right)+D f\left(x_{k}\right)[\xi]+\frac{1}{2} D^{2} f\left(x_{k}\right)[\xi, \xi] \\
& \text { s.t. }\langle\xi, \xi\rangle \leq \Delta_{k}^{2},
\end{aligned}
$$

where $D F\left(x_{k}\right)[z]$ denotes the directional derivative of the function $F$ at $x_{k}$ in the direction of $z$ and $\Delta_{k}$ is the trustregion radius. The quality of the model $m$ is evaluated by means of the quotient

$$
\rho_{k}=\frac{f\left(x_{k}\right)-f\left(x_{k}+\xi\right)}{m(0)-m(\xi)} .
$$

If $\rho$ is close to 0 or negative, then the model is very inaccurate; i.e., the step must be rejected, and the trustregion radius must be reduced. If $\rho$ is larger but still small, the step is accepted, and the trust-region radius is reduced. Finally, if $\rho$ is close to 1 , then there is a good correspondence between the model and the cost function; the step is accepted, and the trust-region radius can be increased.

In the Riemannian trust-region method, at the $k$-th iteration $X^{(k)}$, by utilizing the Taylor expansion on manifold, we consider the following trust-region subproblem on the tangent space:

$$
\begin{array}{ll}
\min _{\xi \in T_{X}^{(k)} \mathrm{St}(n, p)} & m_{X^{(k)}}(\xi)=f\left(X^{(k)}\right)+\left\langle\operatorname{grad} f\left(X^{(k)}\right), \xi\right\rangle+\frac{1}{2}\left\langle\operatorname{Hess} f\left(X^{(k)}\right)[\xi], \xi\right\rangle \\
\text { s.t. } & \sqrt{\langle\xi, \xi\rangle} \leq \Delta^{(k)} .
\end{array}
$$

From (47) and (48), we have

$$
\begin{aligned}
\left\langle\text { Hess } f\left(X^{(k)}\right)[\xi], \xi\right\rangle & =\left\langle X^{(k)} U_{H}^{(k)}+X_{\perp}^{(k)} V_{H}^{(k)}, X^{(k)} U+X_{\perp}^{(k)} V\right\rangle \\
& =\operatorname{tr}\left(U_{H}^{(k)} T U\right)+\operatorname{tr}\left(V_{H}^{(k)} T V\right) .
\end{aligned}
$$

On the other hand, since $\operatorname{grad} f\left(X^{(k)}\right) \in T_{X^{(k)}} \operatorname{St}(n, p)$, then from Lemma 3 , grad $f(X)$ can also be uniquely expressed as $\operatorname{grad} f\left(X^{(k)}\right)=X^{(k)} U_{g}^{(k)}+X_{\perp}^{(k)} V_{g}^{(k)}$ with $U_{g}^{(k)}=X^{(k)^{T}} \operatorname{grad} f\left(X^{(k)}\right)$ and $V_{g}^{(k)}=X_{\perp}^{(k)} \operatorname{Tgrad} f\left(X^{(k)}\right)$. And

$$
\left\langle\operatorname{grad} f\left(X^{(k)}\right)[\xi], \xi=\left\langle X^{(k)} U_{g}^{(k)}+X_{\perp}^{(k)} V_{g}^{(k)}, X^{(k)} U+X_{\perp}^{(k)} V\right\rangle=\operatorname{tr}\left(U_{g}^{(k) T} U\right)+\operatorname{tr}\left(V_{g}^{(k) T} V\right)\right\rangle
$$

Then, the Riemannian trust-region subproblem (77) can be written as

$$
\begin{array}{ll}
\min _{U, V} & m_{X^{(k)}}(U, V)=f\left(X^{(k)}\right)+\operatorname{tr}\left(U_{g}^{(k)} T U+V_{g}^{(k)} T V+\frac{1}{2} U_{H}^{(k)} T U+\frac{1}{2} V_{H}^{(k)} T V\right) \\
\text { s.t. } & \sqrt{\operatorname{tr}\left(U^{T} U\right)+\operatorname{tr}\left(V^{T} V\right)} \leq \Delta^{(k)}, \quad U \in \mathbb{A} \mathbb{S}^{p \times p}, V \in \mathbb{R}^{(n-p) \times p} .
\end{array}
$$


(1) Choose an initial point $X^{(0)} \in \operatorname{St}(n, p)$ and a parameter $\varepsilon_{1}>0$. Set $k$ : $=0$.

(2) while $\left\|\operatorname{grad} f\left(X^{(k)}\right)\right\|_{F}>\varepsilon_{1}$ do

(3) Perform OptStiefelGBB. All parameters needed in OptStiefelGBB are the same as those in [13].

(4) $k:=k+1$.

(5) end while

(6) Set $X^{(0)}:=X^{(k)}$ and $k:=0$.

(7) Perform Steps 2-9 in Algorithm 1.

Algorithm 2: Hybrid method for Problem 1.

In order to provide a guidance for selecting the new trust-region radius $\Delta^{(k)}$, we introduce the quotient

$$
\rho^{(k)}=\frac{f\left(X^{(k)}\right)-f\left(\mathscr{R}_{X^{(k)}}\left(\xi^{(k)}\right)\right)}{m_{X^{(k)}}\left(0_{X^{(k)}}\right)-m_{X^{(k)}}\left(\xi^{(k)}\right)}
$$

which is also used to judge the acceptance or rejection of the candidate $\mathscr{R}_{X^{(k)}}\left(\xi^{(k)}\right)=\mathscr{R}_{X^{(k)}}\left(X^{(k)} U^{(k)}+X_{\perp}^{(k)} V^{(k)}\right)$. Due to the fact that $m_{X^{(k)}}\left(0_{X^{(k)}}\right)=f\left(X^{(k)}\right), \rho^{(k)}$ can be given by

$$
\rho^{(k)}=\frac{2 f\left(\mathscr{R}_{X^{(k)}}\left(\xi^{(k)}\right)\right)-2 f\left(X^{(k)}\right)}{\operatorname{tr}\left(2 U_{g}^{(k)} T U^{(k)}+2 V_{g}^{(k)} T V^{(k)}+U_{H}^{(k)} T U^{(k)}+V_{H}^{(k)} T V^{(k)}\right)} .
$$

As in the Euclidean space setting, the constants (1/4) and $(3 / 4)$ are compared with the ratio $\rho^{(k)}$ and the result determines the trust-region radius in the next iteration. Except that, the constant $\rho^{\prime} \in[0,(1 / 4))$ is used to measure $\rho^{(k)}$. The trust-region step will be taken as the next iteration if $\rho^{(k)}>\rho^{\prime}$, and rejected, otherwise. Specifically, we present the Riemannian trust-region method for Problem 1 as follows.

The trust-region subproblem (80) is solved iteratively and forms the inner iteration of Algorithm 3. Note that (80) is a minimization problem in $\mathbb{A} \mathbb{S}^{p \times p} \times \mathbb{R}^{(n-p) \times p}$ and could then be solved by many classical approaches. A widely used approach is the truncated conjugate gradient algorithm (tCG) due to the following reasons:

(1) The tCG is a Krylov subspace-based solver in which if the initial guess $U^{0} \in \mathbb{A} \mathbb{S}^{p \times p}$ and $V^{0} \in \mathbb{R}^{(n-p) \times p}$, then the sequences $\left\{U^{j}\right\}$ and $\left\{V^{j}\right\}$ generated by tCG always satisfy $U^{j} \in \mathbb{A} \mathbb{S}^{p \times p}$ and $V^{j} \in \mathbb{R}^{(n-p) \times p}$, and hence the approximation $\xi^{j}=X^{(k)} U^{j}+X_{\perp}^{(k)} V^{j} \in$ $T_{X^{(k)}} \operatorname{St}(n, p)$.

(2) The sequence $\left\{m_{X^{(k)}}\left(U^{j}, V^{j}\right)\right\}$ is strictly decreasing while $\operatorname{tr}\left(\left(U^{j}\right)^{T} U^{j}\right)+\operatorname{tr}\left(\left(V^{j}\right)^{T} V^{j}\right)$ is strictly increasing, which makes the RTR a descent approach and guarantees the global convergence (see [[24], Proposition 7.3.2.]).

The pesudocode of tCG is presented in Algorithm 3 for completeness. Note that we use indices in superscript without round brackets to denote the evolution of $U$ and $V$ within the inner iteration, while superscripts with round brackets are used in the outer iteration. As suggested by Absil et al. [24], the algorithm can stop in either after a fixed number of iterations or by the criterion:

$$
\begin{aligned}
& \sqrt{\operatorname{tr}\left(\left(R_{U}\right)_{j}^{T}\left(R_{U}\right)_{j}\right)+\operatorname{tr}\left(\left(R_{V}\right)_{j}^{T}\left(R_{V}\right)_{j}\right)} \\
& \leq \max \left(\left\|R_{0}\right\|_{F} \min \left(\left\|R_{0}\right\|_{F}^{\theta}, \kappa\right)\right),
\end{aligned}
$$

where $\kappa, \theta>0$ are real parameters and $\left\|R_{0}\right\|_{F}=$ $\sqrt{\operatorname{tr}\left(\left(R_{U}\right)_{0}^{T}\left(R_{U}\right)_{0}\right)+\operatorname{tr}\left(\left(R_{V}\right)_{0}^{T}\left(R_{V}\right)_{0}\right)}$. In our numerical testing, we set $\kappa=0.1$ and $\theta=1$.

Remark 5. Fix $k$, and let $\xi^{j} \in T_{X^{(k)}} \operatorname{St}(n, p)$ be the $j$ th iterate of the tCG method for the subproblem expressed by (77). In the existing RTR method, which directly solves the subproblem (77), $\xi^{j}$ is updated as a tangent vector in $T_{X^{(k)}} \operatorname{St}(n, p)$, while in Algorithm 4 , we update $U^{j} \in \mathbb{A} \mathbb{S}^{p \times p}$ and $V^{j} \in \mathbb{R}^{(n-p) \times p}$, where $\xi^{j}=X^{(k)} U^{j}+X_{\perp}^{(k)} V^{j}$. That is, if the tCG method for (77) terminates with $U^{j}$ and $V^{j}$, we only have to compute $\xi^{j}=X^{(k)} U^{j}+X_{\perp}^{(k)} V^{j}$. We do not have to compute $\xi^{i}$ for $i<j$. Furthermore, in the existing RTR method, hess $f\left(X^{(k)}\right)\left[\delta_{j}\right]$ for some $\delta_{j}=X^{(k)} M_{j}+X_{\perp}^{(k)} N_{j}$ $\in T_{X^{(k)}} \operatorname{St}(n, p)$ must to computed at each iteration of the tCG method. However, the proposed Algorithm 4 needs only $\left(M_{H}\right)_{j} \in \mathbb{A} \mathbb{S}^{p \times p}$ and $\left(N_{H}\right)_{j} \in \mathbb{R}^{(n-p) \times p}$, where hess $f\left(X^{(k)}\right)\left[\delta_{j}\right]=X^{(k)}\left(M_{H}\right)_{j}+X_{\perp}^{(k)}\left(N_{H}\right)_{j}$. Therefore, if the number of iterations in the inner tCG method needed for solving the trust-region subproblems is sufficiently large, the proposed Algorithm 4 may have a shorter total computational time than the existing RTR method which directly solves the subproblem (77). These facts imply that our proposed method can reduce the computational cost.

The convergence of the general RTR-tCG on a smooth Riemannian manifold has been extensively analyzed in $[24,37]$ where both the global convergence and local convergence rate have been established under appropriate assumptions. Therefore, to understand the performance of the RTR-tCG method for solving Problem 1, we only need to check these assumptions, which leads to the following conclusions.

Theorem 2 (global convergence). Let $\left\{X^{(k)}\right\}$ be a sequence of iterates generated by Algorithms 3 and 4 , and let an iterate be accepted if $\rho^{k}>\rho^{\prime}$ with $\rho^{\prime} \in(0,(1 / 4))$. Then,

$$
\lim _{k \longrightarrow \infty} \operatorname{grad} f\left(X^{(k)}\right)=0 \text {. }
$$

In fact, this conclusion is a straightforward result of Theorem 4.4 and Corollary 4.6 of [37], based on the fact that 
(1) Choose parameters $\bar{\Delta}>0, \Delta_{0} \in(0, \bar{\Delta}), \rho^{\prime} \in[0,(1 / 4))$, and an initial point $X^{(0)} \in \operatorname{St}(n, p)$.

(2) for $k=0,1,2, \ldots$ do

(3) Obtain $\xi^{(k)}=X^{(k)} U^{(k)}+X_{\perp}^{(k)} V^{(k)}$ by solving the trust-region subproblem (80).

(4) Evaluate $\rho^{(k)}$ from (82).

(5) if $\rho^{(k)}<(1 / 4)$ then

(6) $\Delta^{(k+1)}=(1 / 4) \Delta^{(k)}$.

(7) else if $\rho^{(k)}>(3 / 4)$ and $\left\|\xi^{(k)}\right\|_{F}=\Delta^{(k)}$ then

(8) $\quad \Delta^{(k+1)}=\min \left(2 \Delta^{(k)}, \bar{\Delta}\right)$.

(9) else

(10) $\Delta^{(k+1)}=\Delta^{(k)}$.

(11) end if

(12) if $\rho^{(k)}>\rho^{\prime}$ then

(13) $\quad X^{(k+1)}=\mathscr{R}_{X^{(k)}}\left(\xi^{(k)}\right)$.

(14) else

(15) $\quad X^{(k+1)}=X^{(k)}$.

(16) end if

(17) end for

Algorithm 3: Riemannian trust-region method for Problem 1.

(1) Initialization: set $U^{0}=0 \in \mathbb{A} \mathbb{S}^{p \times p}$ and $V^{0}=0 \in \mathbb{R}^{(n-p) \times p} ;\left(R_{U}\right)_{0}=U_{g}^{(k)}$ and $\left(R_{V}\right)_{0}=V_{g}^{(k)}$; and $M_{0}=-\left(R_{U}\right)_{0}$ and $N_{0}=-\left(R_{V}\right)_{0}$.

(2) for $j=0,1,2, \ldots$ do

(3) if $\operatorname{tr}\left(\left(M_{H}\right)_{j}^{T} M_{j}\right)+\operatorname{tr}\left(\left(N_{H}\right)_{j}^{T} N_{j}\right) \leq 0$ (negative curvature) then

(4) Compute $\tau$ such that $U=U^{j}+\tau M_{j}$ and $V=V^{j}+\tau N_{j} \quad$ minimize $m_{X^{(k)}}(U, V) \quad$ in $\quad(80) \quad$ and $\quad$ satisfy $\sqrt{\operatorname{tr}\left(U^{T} U\right)+\operatorname{tr}\left(V^{T} V\right)}=\Delta^{(k)}$.

(5) Return $U$ and $V$.

(6) else

(7) Set $\alpha_{j}=\left(\left(\operatorname{tr}\left(\left(R_{U}\right)_{j}^{T}\left(R_{U}\right)_{j}\right)+\operatorname{tr}\left(\left(R_{V}\right)_{j}^{T}\left(R_{V}\right)_{j}\right)\right) /\left(\operatorname{tr}\left(\left(M_{H}\right)_{j}^{T} M_{j}\right)+\operatorname{tr}\left(\left(N_{H}\right)_{j}^{T} N_{j}\right)\right)\right)$.

(8) Set $U^{j+1}=U^{j}+\alpha_{j} M_{j}$ and $V^{j+1}=V^{j}+\alpha_{j} N_{j}$.

(9) if $\sqrt{\operatorname{tr}\left(\left(U^{j+1}\right)^{T} U^{j+1}\right)+\operatorname{tr}\left(\left(V^{j+1}\right)^{T} V^{j+1}\right)} \geq \Delta^{(k)}$ (exceeded trust-region) then

(10) Compute $\tau \geq 0$ such that $U=U^{j}+\tau M_{j}$ and $V=V^{j}+\tau N_{j}$ satisfy $\sqrt{\operatorname{tr}\left(U^{T} U\right)+\operatorname{tr}\left(V^{T} V\right)}=\Delta^{(k)}$.

(11) Return $U$ and $V$.

(12) else

(13) Set $\left(R_{U}\right)_{j+1}=\left(R_{U}\right)_{j}+\alpha_{j}\left(M_{H}\right)_{j}$ and $\left(R_{V}\right)_{j+1}=\left(R_{V}\right)_{j}+\alpha_{j}\left(N_{H}\right)_{j}$.

(14) Set $\beta_{j+1}=\left(\left(\operatorname{tr}\left(\left(R_{U}\right)_{j+1}^{T}\left(R_{U}\right)_{j+1}\right)+\operatorname{tr}\left(\left(R_{V}\right)_{j+1}^{T}\left(R_{V}\right)_{j+1}\right)\right) /\left(\operatorname{tr}\left(\left(R_{U}\right)_{j}^{T}\left(R_{U}\right)_{j}\right)+\operatorname{tr}\left(\left(R_{V}\right)_{j}^{T}\left(R_{V}\right)_{j}\right)\right)\right)$.

(15) $\quad$ Set $M_{j+1}=-\left(R_{U}\right)_{j+1}+\beta_{j+1} M_{j}$ and $N_{j+1}=-\left(R_{V}\right)_{j+1}+\beta_{j+1} N_{j}$.

(16) end if

(17) end if

(18) end for

Algorithm 4: The truncated CG (tCG) method for the trust-region subproblem (80).

the cost function $f(X)$ and the adopted retraction as described in Section 2 are smooth, and the involved manifold $\operatorname{St}(n, p)$ is a smooth and compact Riemannian manifold.

Theorem 3 (local convergence speed). Consider Algorithms 3 and 4 with retraction $\mathscr{R}$ as in Section 2 and stopping criterion in Algorithm 4 as in (83). Let $X^{*} \in S t(n, p)$ be a (nondegenerate) local minimum of $f$. Then, there exists $c>0$ such that for all sequences $\left\{X^{(k)}\right\}$ generated by the algorithm converging to $X^{*}$, there exists $K>0$ such that for all $k>K$,

$$
\operatorname{dist}\left(X^{(k+1)}, X^{*}\right) \leq c\left(\operatorname{dist}\left(X^{(k)}, X^{*}\right)\right)^{\min \{\theta+1,2\}},
$$

with $\theta>0$ as in (83), and "dist" defines the Riemannian distance on $S t(n, p)$ (see [24], p. 46).

\section{Numerical Experiments}

In this section, we report the numerical performance of the proposed algorithms for Problem 1. All the numerical experiments were completed on a personal computer with a Intel(R) Core(TM)2 Quad of 2.33 GHz CPU and 3.00 GB of RAM equipped with MATLAB R2019b. We report numerical experiments and application to the least squares fitting of the DEDICOM model and the orthonormal INDSCAL model. To illustrate the efficiency of our algorithms, we also compare Algorithms 2 and 3 with some latest Riemannian conjugate gradient- (CG-) type methods which are all applicable to Problem 1 with necessary modifications and some existing Riemannian second-order algorithms in the MATALB toolbox Manopt. 
5.1. Algorithmic Issues. Throughout our experiments, in the implementation of Algorithm 2, the parameter $\varepsilon_{1}$ in (74) is set to be $\varepsilon_{1}=0.1$. In the implementation of the inner iteration of Algorithm 2, the CR method to the symmetric linear equation (69), the initial matrix is all set to be 0 with suitable size at each repetition of Newton's method, and were stopped when the relative residual 2-norms $\left(\left\|r_{L}^{(k)}\right\|_{2} /\left\|g^{(k)}\right\|_{2}\right)$ became less than $10^{-6}$. Here, the residual is $r_{L}^{(k)}=g^{(k)}-H_{A}^{(k)} x_{L}^{(k)}$, where $x_{L}^{(k)}$ denotes the $L$ th approximation of CR at the $k$ th repetition of Newton's method. The largest number of CR method is set to be 1000. In the implementation of Algorithm 3, OptStiefel is also applied to obtain a suitable initial point, and the parameters are set to be $\Delta_{0}=\sqrt{3}, \rho^{\prime}=0.1$ and $\bar{\Delta}=\infty$.

The stopping criteria for Algorithms 2 and 3 are set to be

$$
\left\|\operatorname{grad} f\left(X^{(k)}\right)\right\|_{F}<\varepsilon,
$$

with $\varepsilon=10^{-6}$. For all the compared Riemannian gradienttype algorithms, we use the same stopping criterion as that in OptStiefelGBB [13]: we let algorithms run up to $K$ iterations and stop it at iteration $k<K$ if $\left\|\operatorname{grad} f\left(X^{(k)}\right)\right\|_{F} \leq \varepsilon$ or $\operatorname{rel}_{X}^{k} \leq \varepsilon_{X}$ and $\operatorname{rel}_{f}^{k} \leq \varepsilon_{f}$ or

$$
\begin{aligned}
& \operatorname{mean}\left\{\operatorname{rel}_{X}^{k-\min \{k, T\}+1}, \ldots, \operatorname{rel}_{X}^{k}\right\} \leq 10 \varepsilon_{X}, \\
& \operatorname{mean}\left\{\operatorname{rel}_{f}^{k-\min \{k, T\}+1}, \ldots, \operatorname{rel}_{f}^{k}\right\} \leq 10 \varepsilon_{f},
\end{aligned}
$$

for some constants $\varepsilon, \varepsilon_{X}, \varepsilon_{f} \in(0,1)$, and $T, K \in \mathbb{N}^{+}$, where

$$
\begin{aligned}
\operatorname{rel}_{X}^{(k)} & =\frac{\left\|X^{(k+1)}-X^{(k)}\right\|_{F}}{\sqrt{p}}, \\
\operatorname{rel}_{f}^{k} & =\frac{\left|f\left(X^{(k+1)}\right)-f\left(X^{(k)}\right)\right|}{\left|f\left(X^{(k)}\right)\right|+1} .
\end{aligned}
$$

The default values of $\varepsilon_{X}, \varepsilon_{f}, T$, and $K$ are $10^{-6}, 10^{-8}, 5$, and 2000, respectively. Throughout, "IT.," "CT.," "Grad.," and "Obj." mean the number of iterations, the total computing time in seconds, the norm of Riemannian gradient grad $f\left(X_{k}\right)$, and the objective function value at the final iterate by implementing the proposed algorithms, respectively.

\subsection{Numerical Experiments and Application to the Least} Squares Fitting of the DEDICOM Model. DEDICOM is a model proposed by Harshman [5] for the analysis of asymmetric data. According to the DEDICOM model, a square data matrix $F$, containing entries $F_{i j}$ representing the (asymmetric) relation of object $i$ to object $j$, is decomposed as

$$
F=X R X^{T}+N
$$

where $X$ is an $n$ by $p(p<n)$ matrix of weights for the $n$ objects on $p$ dimensions or aspects, $R$ is a square matrix of order $p$, representing (asymmetric) relations among the $p$ dimensions, and $N$ is an error matrix with entries $(n / j)$ representing the part of the relation of object $i$ to object $j$ that is not explained by the model. The objective of fitting this model to the data is to explain the data by means of relations among as small a number of dimensions as possible. These dimensions can be considered as "aspects" of the objects. The "loadings" of the objects on these aspects are given by matrix $X$, which is constrained to be columnwise orthonormal. The entries in matrix $X$ indicate the importance of the aspects for the objects. The dimensionality of $R$ and $X$ and hence the number of aspects to be determined are to be based on some external criterion, defined by the user.

The DEDICOM model has to be fit in the least squares sense over matrices $X$ and $R$ of order $n$ by $p$ and $p$ by $p$, respectively. The loss function that is to be minimized can be written as

$$
f(X, R)=\left\|F-X R X^{T}\right\|_{F}^{2}
$$

over matrix $X$ and $R$, subject to the constraint $X^{T} X=I p$. Kiers and ten Berge $[1,2]$ have given an algorithm for this problem based on alternating least squares method that can be interpreted as alternately updating $R$ and $X$. Because $X^{T} X=I_{p}$, the minimum of $f$ over $R$ for fixed $X$ is given by $R=X^{T} F X$. Minimizing $f$ over $X$, for fixed $R$, is equivalent to minimizing

$$
f(X)=c-2 \operatorname{tr}\left(F^{T} X R X^{T}\right), \quad \text { subject to } X^{T} X=I_{p},
$$

which is a particular case of Problem 1 with $A=0, B_{1}=-2 F^{T}$, and $C_{1}=R$. An algorithm based on majorization was provided in [2] for updating this subproblem on $X$. Because majorization algorithm requires a large number of iterations to converge within a given tolerance, we here test numerical performance of our proposed algorithms for updating $X$.

Without loss of generality, the square data matrix $F$ in the DEDICOM model is given by $F=\operatorname{rand} n(n, n)$ and the fixed $R$ is given by $R=\operatorname{rand}(p, p)$. The dimensions $n$ and $p$ were set to 500 and 5 , respectively. The iterations of Algorithms 2 and 3 were started with $X^{(0)}=q f(\operatorname{rand}(n, p))$ or $X^{(0)}=q f\left(X^{*}+\vartheta * \operatorname{rand}(n, p)\right)$, where the parameter $\vartheta$ was set to $10,10^{2}$, and $10^{3}$, and $X^{*}$ is a solution of problem generated by majorization algorithm.

Figure 1 shows the convergence histories of the proposed Algorithm 2 after switching to Newton's method (Algorithm 1), and Algorithm 3 after OptStiefel is performed to generate a suitable initial point for different choices of initial matrix $X^{(0)}$. The number of iterations is plotted on the horizontal axis, and the $\log 10$ of norm of the Riemannian gradient grad $f\left(X^{(k)}\right)$ is plotted on the vertical axis in these figures. More numerical results are reported in Table 2, in which the term "IT." in Algorithms 2 and 3 means the number of iterations required by OptStiefelGBB to satisfy the switching criteria and the number of extra iterations required by Algorithm 2 or Algorithm 3 to achieve the stopping criteria and "Obj." means the objective function value $-2 \operatorname{tr}\left(F^{T} X^{(k)} R X^{(k)^{T}}\right)$ at the final iterate. From Figure 1 and Table 2, we observe that once $X^{(k)}$ arrives in the appropriate region determined by $\varepsilon_{1}$, Algorithms 2 and 3 generate sequences which quickly converge. We can also see that the effectiveness of Algorithms 2 and 3 was not affected by different choices of initial matrix $X^{(0)}$, and the convergence speed of these two algorithm is not very 


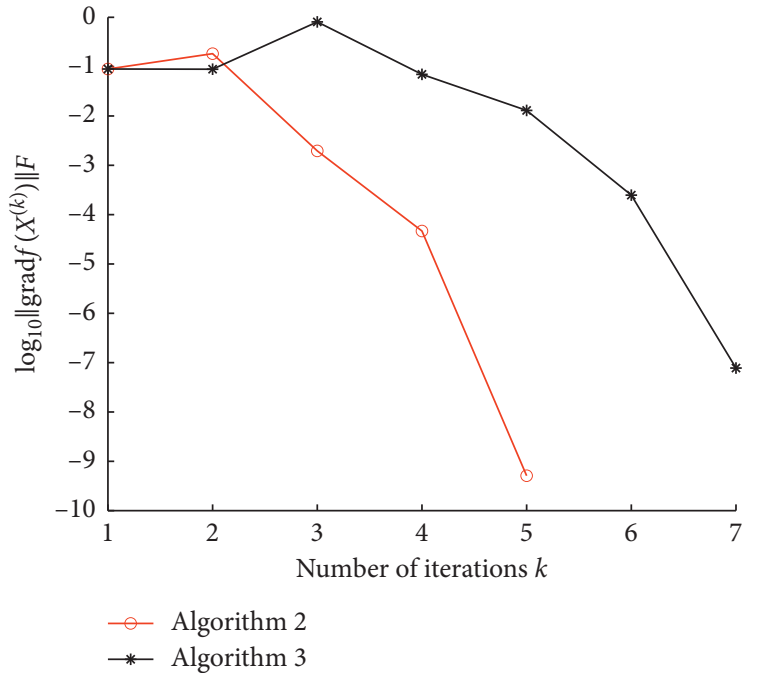

(a)

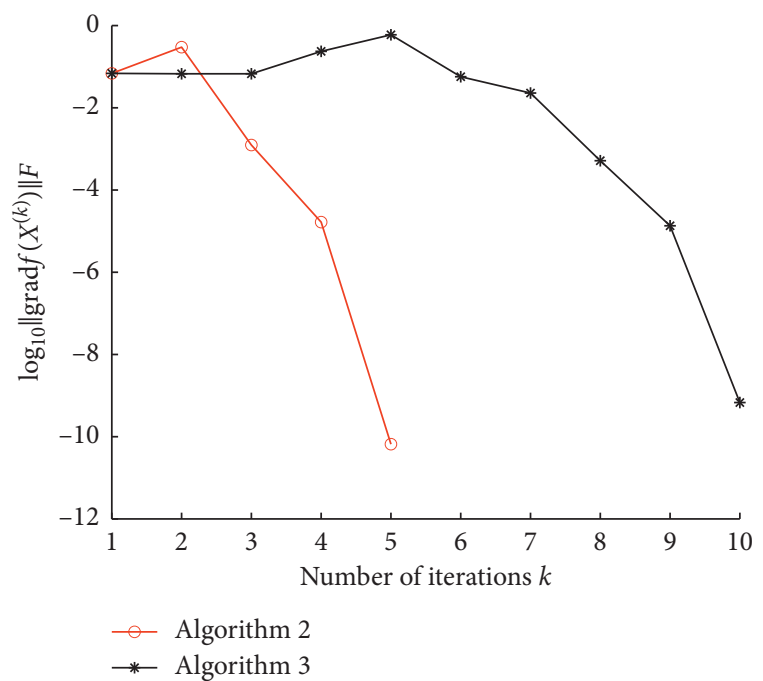

(c)

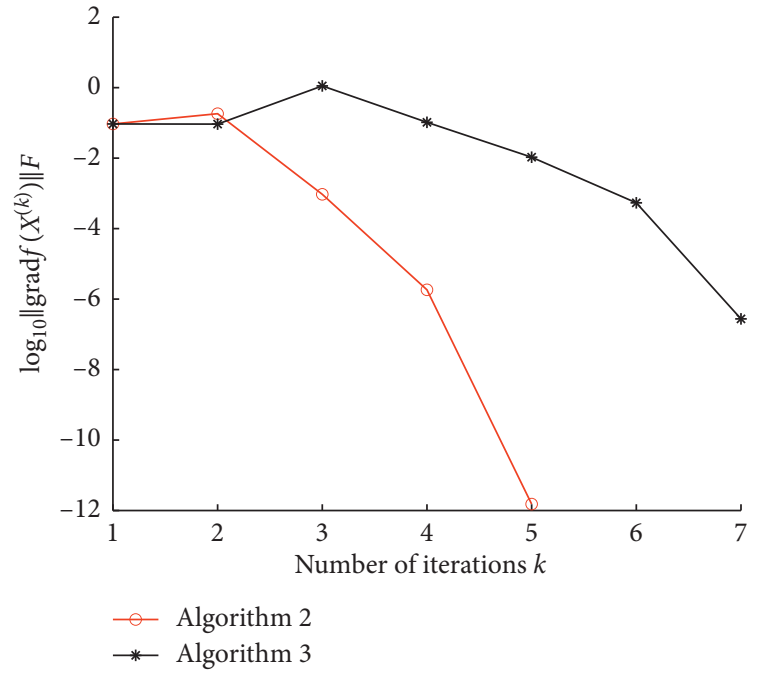

(b)

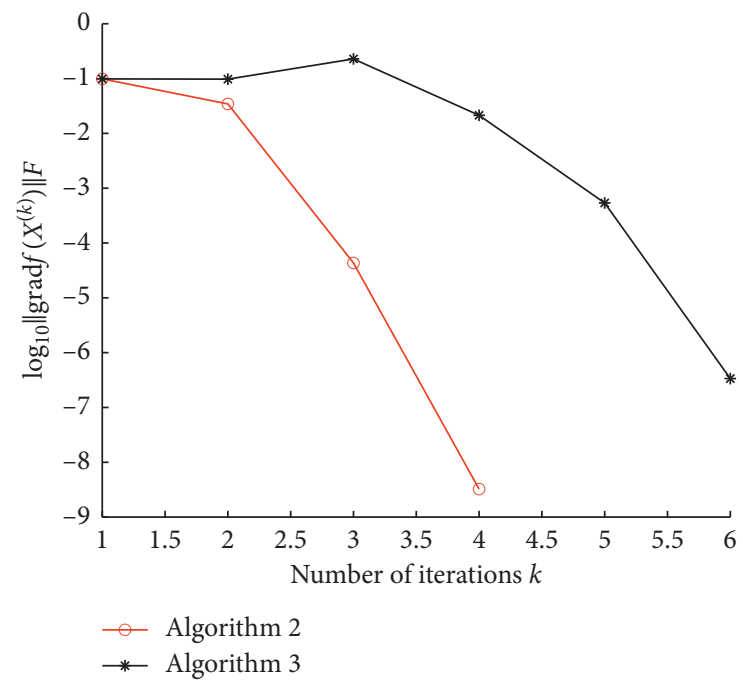

(d)

FIgURE 1: Convergence histories of Algorithm 2 after switching to Newton's method and Algorithm 3 after OptStiefel is performed to generate a suitable initial point, with different initial matrix $X^{(0)}$. (a) $X^{(0)}=q f(\operatorname{rand}(n, p))$. (b) $X^{(0)}=q f\left(X^{*}+10 \mathrm{rand}(n, p)\right)$. (c) $X^{(0)}=q f\left(X^{*}+10^{2} \operatorname{rand}(n, p)\right)$. (d) $X^{(0)}=q f\left(X^{*}+10^{3} \operatorname{rand}(n, p)\right)$.

TABle 2: Numerical results of Algorithms 2 and 3 with different initial matrix $X^{(0)}$.

\begin{tabular}{|c|c|c|c|c|}
\hline & CT. & IT. & Grad. & Obj. \\
\hline \multicolumn{5}{|c|}{$X^{(0)}=\operatorname{rand}(n, p)$} \\
\hline Algorithm 2 & 11.85 & $230 / 4$ & $5.10 \times 10^{-10}$ & -309.19 \\
\hline Algorithm 3 & 1.15 & $230 / 5$ & $7.77 \times 10^{-8}$ & -309.19 \\
\hline \multicolumn{5}{|c|}{$X^{(0)}=q f(\operatorname{rand}(n, p))$} \\
\hline Algorithm 2 & 9.95 & $149 / 4$ & $1.52 \times 10^{-12}$ & -310.24 \\
\hline Algorithm 3 & 0.67 & $149 / 5$ & $2.74 \times 10^{-7}$ & -310.24 \\
\hline \multicolumn{5}{|c|}{$X^{(0)}=q f\left(X^{*}+10^{2} \operatorname{rand}(n, p)\right)$} \\
\hline Algorithm 2 & 11.49 & $189 / 4$ & $6.58 \times 10^{-11}$ & -309.19 \\
\hline Algorithm 3 & 1.50 & $189 / 8$ & $6.77 \times 10^{-10}$ & -309.19 \\
\hline \multicolumn{5}{|c|}{$X^{(0)}=q f\left(X^{*}+10^{3} \operatorname{rand}(n, p)\right)$} \\
\hline Algorithm 2 & 6.41 & $264 / 3$ & $3.24 \times 10^{-9}$ & -310.24 \\
\hline Algorithm 3 & 0.62 & $264 / 4$ & $3.36 \times 10^{-7}$ & -310.24 \\
\hline
\end{tabular}


sensitive to the choice of $X^{(0)}$. This is in accordance with the global convergence of these two algorithms established in Proposition 3 and Theorem 2, respectively. For this reason, we use $X^{(0)}=q f(\operatorname{rand}(n, p))$ in the subsequent examples.

Next, we also show the detailed numerical results when applying the CR method to the symmetric linear equation (69) directly in the implementation of step 7 of Algorithm 2. Figure 2 shows the convergence histories of the CR method for (69). The number of iterations of the CR method is plotted on the horizontal axis, and the $\log 10$ of the relative residual norm, $\left(\left\|r_{L}^{(k)}\right\|_{2} /\left\|g^{(k)}\right\|_{2}\right)$, is plotted on the vertical axis in the figures. More numerical results are reported in Table 3. We can observe from Figure 2 and Table 3 that the CR method is effective for solving the Newton equation (69) as the symmetric linear system, and that Newton's method, (69), generates locally quadratically convergent sequences.

Finally, we numerically compare Algorithm 3 in which the modified trust-region subproblems (80) are solved by Algorithm 4 with the existing trust-region method (denoted by Existing RTR) in MATLAB toolbox Manopt [38]. We fix $n=500$. For each $p \in\{5,10, \ldots, 50\}$, we compute the total computing time needed for convergence. Table 4 and Figure 3 show that the performance of the proposed Algorithm 3 is slightly better than that of the existing method in terms of computational time. This phenomenon becomes more pronounced as the matrix dimension increases.

\subsection{Numerical Comparison with Latest Riemannian CG} Methods for the Least Squares Fitting of the Orthonormal INDSCAL Model. The orthonormal INDSCAL model is used to consider a three-way array consisting of $m$ symmetric $n \times n$ slices $S_{j}[7,8,10]$. In the model, these slices $S_{j}$ are composed by the doubly centered dissimilarity matrices. The orthonormal INDSCAL model decomposes each slice as

$$
S_{j}=X D_{j} X^{T}+E_{j} \text {, }
$$

where $X$ is a $n \times r$ matrix (assumed to be columnwise orthonormal), $D_{j}$ is a diagonal matrix, and $E_{j}$ is a $n \times n$ matrix, containing the errors of the model fit. That means all slices share a common loading matrix $X$ and differ from each other only by the diagonal elements of $D_{j}$ called idiosyncratic saliences. The orthonormal INDSCAL model, appeared initially in psychometric literature, is now widely used in social sciences, marketing research, etc.

The orthonormal INDSCAL problem seeks for $\left(X, D_{1}, D_{2}, \ldots, D_{m}\right)$ such that the model fits the data in least squares sense, i.e., for given and fixed $n \times n$ symmetric matrices $S_{j}, j=1,2, \ldots, m$, minimizes the function:

$$
\begin{array}{ll}
\text { minimize } & f\left(X, D_{j}\right)=\sum_{i=1}^{m}\left\|S_{i}-X D_{i} X^{T}\right\|_{F}^{2} \\
\text { subject to } & \left(X, D_{1}, D_{2}, \ldots, D_{m}\right) \in \operatorname{St}(n, r) \times \mathbb{D}(r)^{m},
\end{array}
$$

where $\mathbb{D}(r)^{m}=\mathbb{D}(r) \times \cdots \times \mathbb{D}(r)$ and $\mathbb{D}(r)$ denotes the set of all $r \times r$ diagonal matrices. This optimization problem has no direct analytical solution. The standard numerical solution of this problem is given by an alternating least squares algorithm $[1,8,10]$. Minimizing $f$ over $X$, for fixed $D_{j}$, $j=1,2, \ldots, m$, is equivalent to minimizing

$$
f(X)=c-2 \sum_{j=1}^{m} \operatorname{tr}\left(S_{j} X D_{j} X^{T}\right), \quad \text { subject to } X^{T} X=I_{r},
$$

which is also a particular case of Problem 1 with $A=0$, $B_{j}=-2 S_{j}, C_{j}=D_{j}, j=1, \ldots, m$. A majorization-based algorithm was provided in [1] for updating this subproblem on $X$. Here, we test our proposed algorithms for this $X$ subproblem.

To illustrate the efficiency of our algorithms, we also compare them with the existing Riemannian trust-region algorithm (denoted by existing RTR) and the existing Riemannian BFGS algorithm (denoted by existing RBFGS) in MATLAB toolbox Manopt [38] and some latest Riemannian conjugate gradient- (CG-) type methods which are all applicable with necessary modifications. To this end, we first introduce the iterative framework of each compared Riemannian CG method. For solving Problem 1, update the iterated using the following scheme starting in $X_{0} \in \operatorname{St}(n, p)$ with $\xi_{0}=-\operatorname{grad} f\left(X^{(0)}\right)$,

$$
X^{(k+1)}=\mathscr{R}\left(t_{k} \xi^{(k)}\right),
$$

where $t_{k}>0$ is the step size and $\xi^{(k)} \in \mathbf{T}_{X} \operatorname{St}(n, p)$ is

$$
\xi^{(k+1)}=-\operatorname{grad} f\left(X^{(k)}\right)+\beta_{k+1} \mathscr{T}_{t_{k} \xi^{(k)}}\left(\xi^{(k)}\right),
$$

where $\mathscr{T}$ is a vector transport on $\operatorname{St}(n, p)$ [24], which is a smooth mapping from the product of tangent bundles $\mathbf{T}_{X} \mathrm{St}(n, p) \oplus \mathbf{T}_{X} \mathrm{St}(n, p)$ to the tangent bundle $\mathbf{T}_{X} \mathrm{St}(n, p)$, where $\oplus$ is the Whitney sum. There are several expressions to update the parameter $\beta_{k+1}$ of equation (1), and some of the most popular expressions are $\beta$ of the Fletcher-Reeves method:

$$
\beta_{k+1}^{\mathrm{FR}}=\frac{\left\|\operatorname{grad} f\left(X^{(k+1)}\right)\right\|_{F}^{2}}{\left\|\operatorname{grad} f\left(X^{(k)}\right)\right\|_{F}^{2}},
$$

and $\beta$ of the Polak-Ribière method:

$$
\beta_{k+1}^{\mathrm{PR}}=\frac{\left\langle\operatorname{grad} f\left(X^{(k+1)}\right), \operatorname{grad} f\left(X^{(k+1)}\right)-\mathscr{T}_{t_{k} \xi^{(k)}}\left(\operatorname{grad} f\left(X^{(k)}\right)\right)\right\rangle}{\left\|\operatorname{grad} f\left(X^{(k)}\right)\right\|_{F}^{2}} .
$$

In this experiment, we compare Algorithms 2 and 3 with the following Riemannian CG methods. We note that the differences between these Riemannian CG methods are mainly due to their specific ways of updating the parameters $t_{k}$ and $\beta_{k+1}$ and constructing retractions and vector transports.

(i) "RGPRP-CG": the Riemannian geometric Polak-Ribière-Polay CG method used in [16] designed to solve Problem 1. The retraction adapted is the QR-based retraction (19), and the vector transport is constructed by using the orthogonal projection operator onto the tangent space, which has the form 


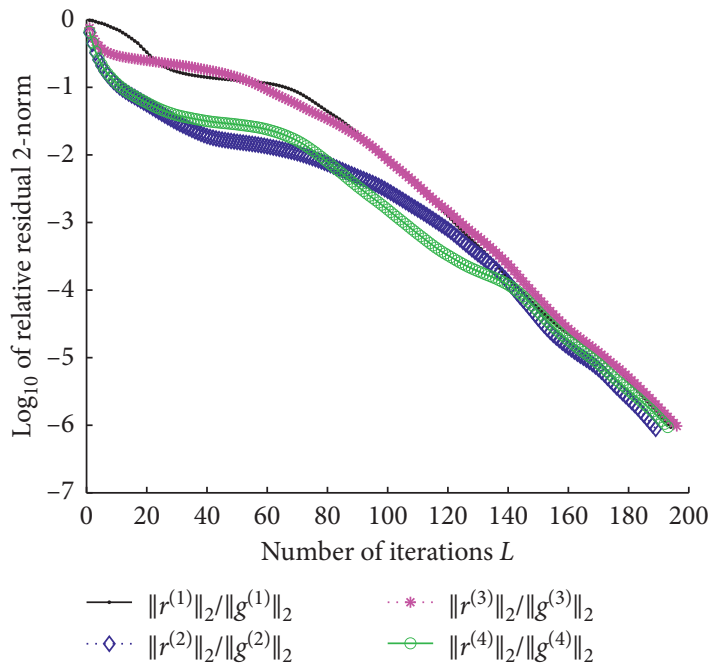

(a)

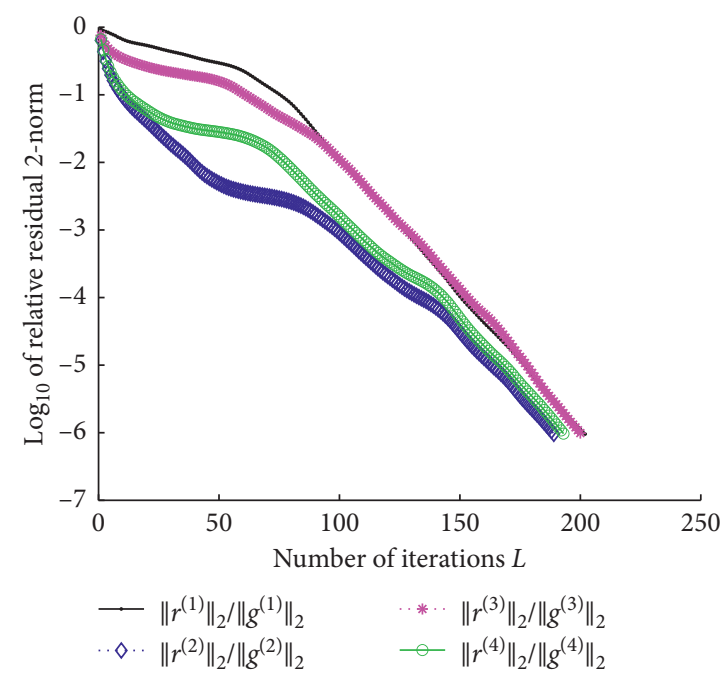

(c)

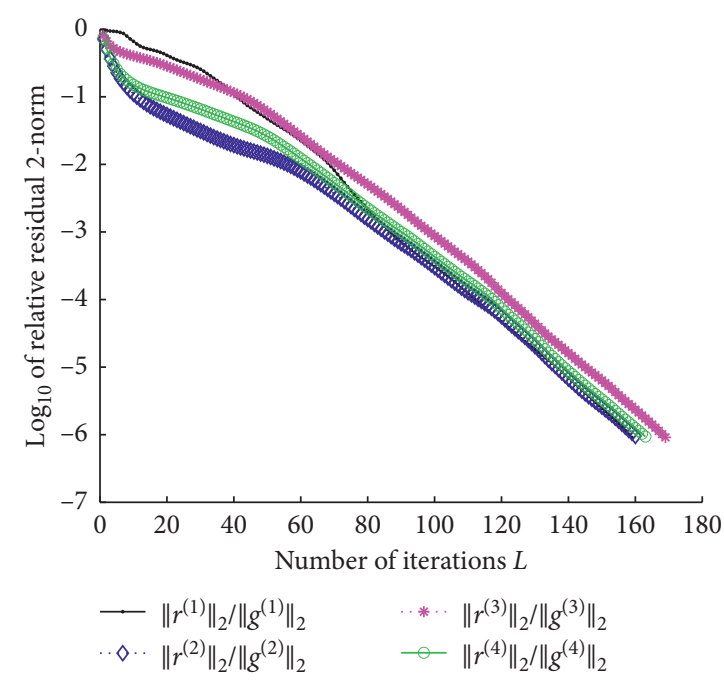

(b)

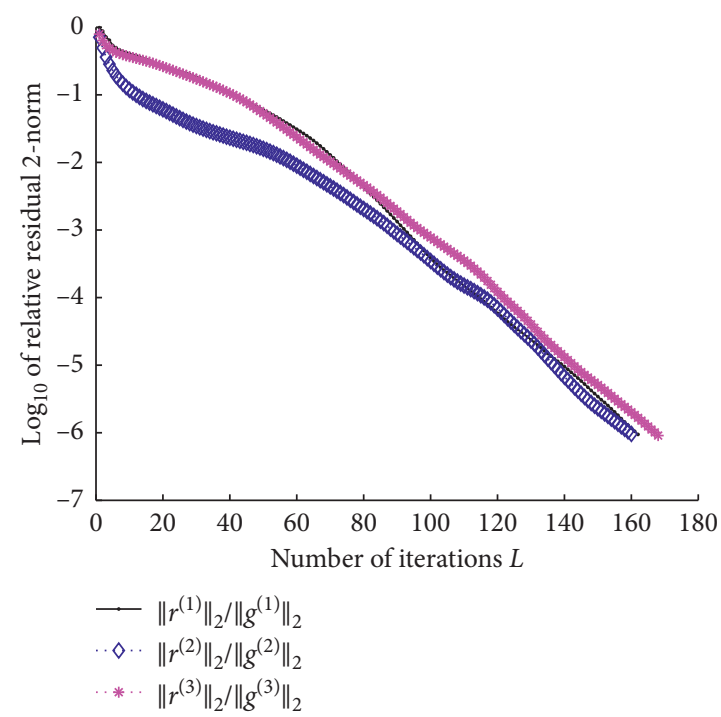

(d)

Figure 2: Convergence histories of the CR method to the Newton equation (69), with different initial matrix $X^{(0)}$ in Algorithm 2. (a) $X^{(0)}=q f(\operatorname{rand}(n, p))$. (b) $X^{(0)}=q f\left(X^{*}+10 \operatorname{rand}(n, p)\right)$. (c) $X^{(0)}=q f\left(X^{*}+10^{2} \operatorname{rand}(n, p)\right)$. (d) $X^{(0)}=q f\left(X^{*}+10^{3} \operatorname{rand}(n, p)\right)$.

$$
\begin{aligned}
\mathscr{T}_{t_{k} \xi^{(k)}}\left(\xi^{(k)}\right)= & \mathbf{P}_{\mathscr{R}_{X}(k)}\left(t_{k} \xi^{(k)}\right) \\
= & \xi^{(k)}-q f\left(\xi^{(k)}\right) \\
& \cdot \operatorname{sym}\left(q f\left(X^{(k)}+t_{k} \xi^{(k)} \xi^{(k)}\right)^{T} \xi^{(k)}\right) .
\end{aligned}
$$

The linear search is the Armijo-type line search and the CG direction $\xi_{k+1}$ is given by

$$
\xi^{(k+1)}=-\operatorname{grad} f\left(X^{(k+1)}\right)+\beta_{k+1} \mathscr{T}_{\alpha_{k} \xi^{(k)}}\left(\xi^{(k)}\right)-\theta_{k+1} Y_{k} \text {, }
$$

where

$$
Y_{k}=\operatorname{grad} f\left(X^{(k+1)}\right)-\mathscr{T}_{\alpha_{k} \xi^{(k)}} \operatorname{grad} f\left(X^{(k)}\right),
$$

with $\beta_{k+1}$ as in (3) and $\theta_{k+1}=\left(\left\langle\operatorname{grad} f\left(X^{(k+1)}\right)\right.\right.$, $\left.\mathscr{T}_{\alpha_{k} \xi^{(k)}} \xi^{(k)}>/\left\|\operatorname{grad} f\left(X^{(k)}\right)\right\|_{F}^{2}\right)$.

(ii) "RFR-CG": the Riemannian Fletcher-Reeves CG method used in [18] designed to solve Problem 1, with the same QR-based retraction and vector transport as "GPRP." The step size is determined by $t_{k}=\max \left\{\bar{t} \rho^{j}, j=0,1,2, \ldots\right\}$ such that

$$
\begin{aligned}
& f\left(\mathscr{R}_{X^{(k)}}\left(t_{k} \xi^{(k)}\right)\right)-f\left(X^{(k)}\right) \\
& \quad \leq \delta_{1} t_{k}\left\langle\operatorname{grad} f\left(X^{(k)}\right), \xi^{(k)}\right\rangle-\delta_{2} t_{k}^{2}\left\|\xi^{(k)}\right\|_{F}^{2},
\end{aligned}
$$

where $\bar{t}$ is the initial step length, $\delta_{1} \in(0,1)$, and $\delta_{2}>0$. The CG direction $\xi^{(k+1)}$ is given as 
TABLE 3: Numerical results of the CR method to the symmetric linear equation (69) with different initial matrix $X^{(0)}$ in Algorithm 2.

\begin{tabular}{|c|c|c|c|}
\hline$L$ & CT. & IT. & $\left(\left\|r_{L}^{(k)}\right\|_{2} /\left\|g^{(k)}\right\|_{2}\right)$ \\
\hline \multicolumn{4}{|c|}{$X^{(0)}=q f(\operatorname{rand}(n, p))$} \\
\hline 1 & 1.98 & 194 & $9.38 \times 10^{-7}$ \\
\hline 2 & 2.05 & 189 & $8.86 \times 10^{-7}$ \\
\hline 3 & 1.99 & 196 & $9.74 \times 10^{-7}$ \\
\hline 4 & 2.14 & 193 & $9.39 \times 10^{-7}$ \\
\hline \multicolumn{4}{|c|}{$X^{(0)}=q f\left(X^{*}+10 \operatorname{rand}(n, p)\right)$} \\
\hline 1 & 1.78 & 159 & $9.08 \times 10^{-7}$ \\
\hline 2 & 1.63 & 160 & $9.56 \times 10^{-7}$ \\
\hline 3 & 1.83 & 169 & $9.13 \times 10^{-7}$ \\
\hline 4 & 1.63 & 163 & $9.38 \times 10^{-7}$ \\
\hline \multicolumn{4}{|c|}{$X^{(0)}=q f\left(X^{*}+10^{2} \operatorname{rand}(n, p)\right)$} \\
\hline 1 & 2.02 & 202 & $9.56 \times 10^{-7}$ \\
\hline 2 & 2.02 & 189 & $9.61 \times 10^{-7}$ \\
\hline 3 & 2.03 & 200 & $9.97 \times 10^{-7}$ \\
\hline 4 & 2.12 & 193 & $9.69 \times 10^{-7}$ \\
\hline \multicolumn{4}{|c|}{$X^{(0)}=q f\left(X^{*}+10^{3} \operatorname{rand}(n, p)\right)$} \\
\hline 1 & 1.66 & 162 & $9.45 \times 10^{-7}$ \\
\hline 2 & 1.79 & 160 & $9.61 \times 10^{-7}$ \\
\hline 3 & 1.71 & 168 & $9.16 \times 10^{-7}$ \\
\hline
\end{tabular}

TABle 4: Computational time (seconds) between Algorithm 3 and the existing trust-region method.

\begin{tabular}{lcccccccccc}
\hline$p$ & 5 & 10 & 15 & 20 & 25 & 30 & 35 & 40 & 45 & 50 \\
\hline Algorithm 3 & 2.80 & 3.66 & 4.98 & 9.73 & 20.63 & 12.66 & 28.29 & 22.46 & 35.30 & 38.47 \\
Existing RTR & 3.80 & 6.39 & 10.83 & 22.17 & 27.66 & 26.01 & 43.25 & 49.15 & 67.54 & 72.38 \\
\hline
\end{tabular}

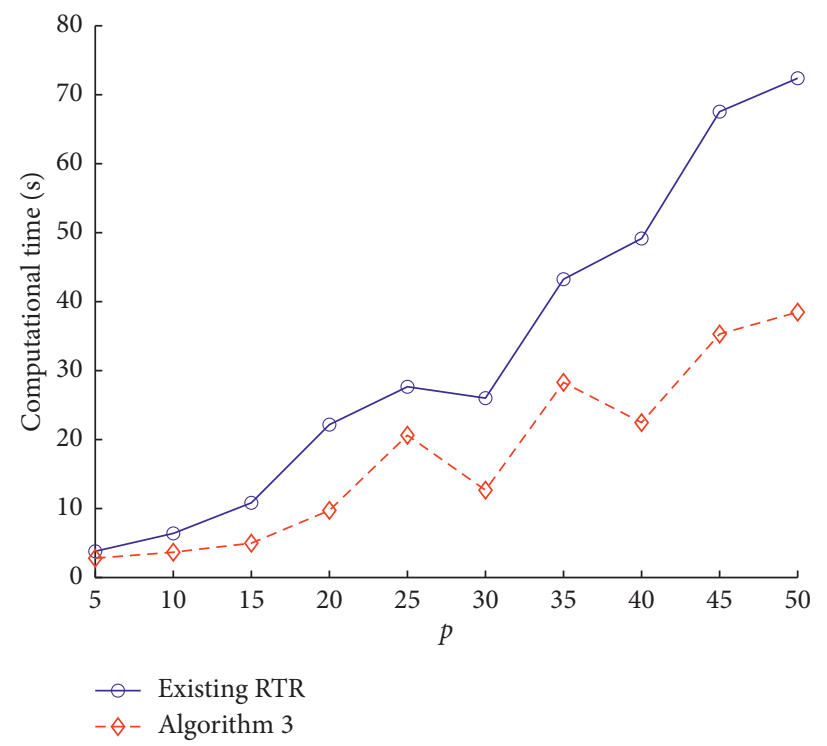

Figure 3: Computational time (seconds) of Algorithm 3 and the existing trust-region method.

$$
\begin{aligned}
\xi^{(k+1)}= & -\operatorname{grad} f\left(X^{(k+1)}\right)+\beta_{k+1} \mathscr{T}_{\alpha_{k} \xi^{(k)}}\left(\xi^{(k)}\right) \\
& -\theta_{k+1} \operatorname{grad} f\left(X^{(k+1)}\right),
\end{aligned}
$$

with $\beta_{k+1}$ as in $(2)$ and $\theta_{k+1}=\left(\left\langle\operatorname{grad} f\left(X^{(k+1)}\right)\right.\right.$, $\left.\left.\xi^{(k+1)}\right\rangle /\left\|\operatorname{grad} f\left(X^{(k)}\right)\right\|_{F}^{2}\right)$. (iii) "RCG-Cayley": the Riemannian case of Dai's nonlinear CG method used in [14] designed to solve Problem 1 by using the Cayley transformation (17) to construct the retraction and the differentiated retraction to construct the vector transport

$$
\mathscr{T}_{t_{k} \xi^{(k)}}\left(\xi^{(k)}\right)=\left(I_{n}-\frac{t_{k}}{2} W_{\xi^{(k)}}\right)^{-2} \xi^{(k)}
$$



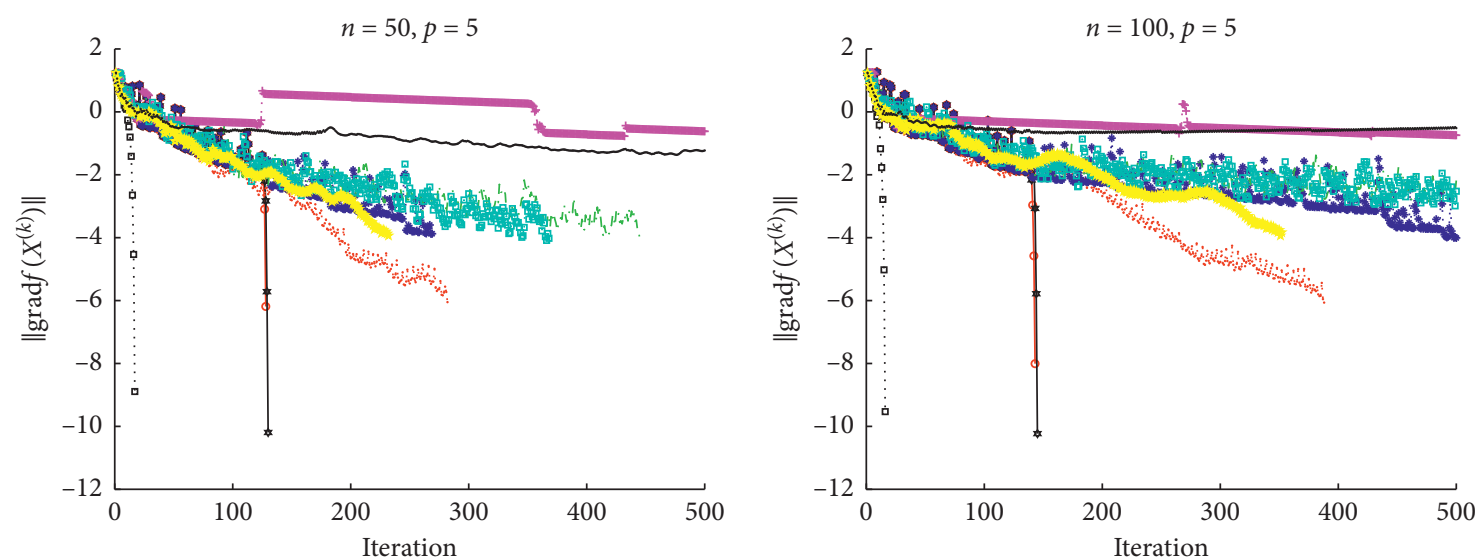

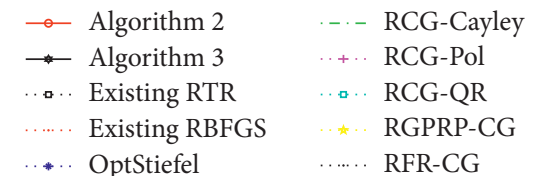

(a)

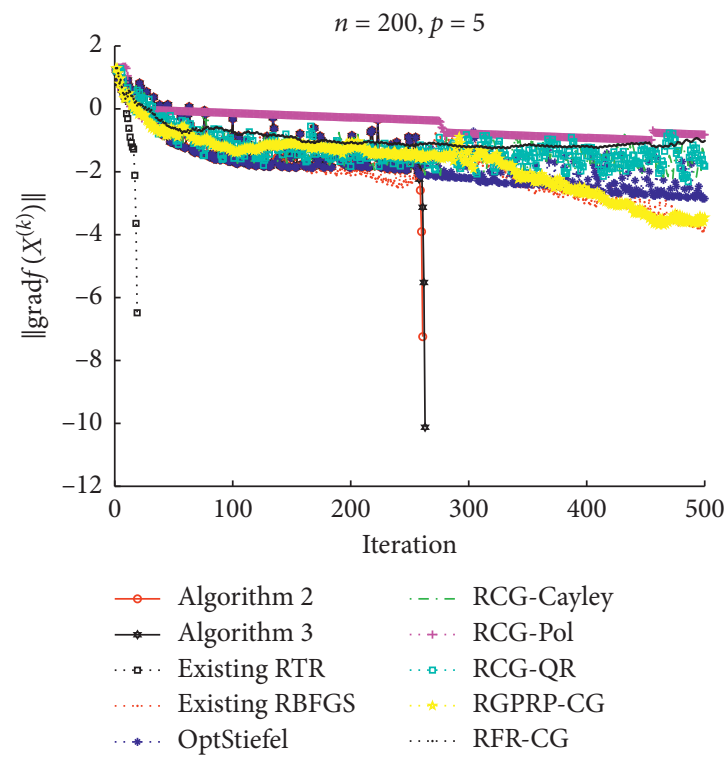

(c)

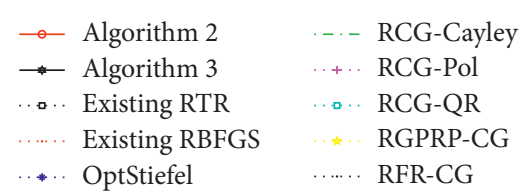

(b)

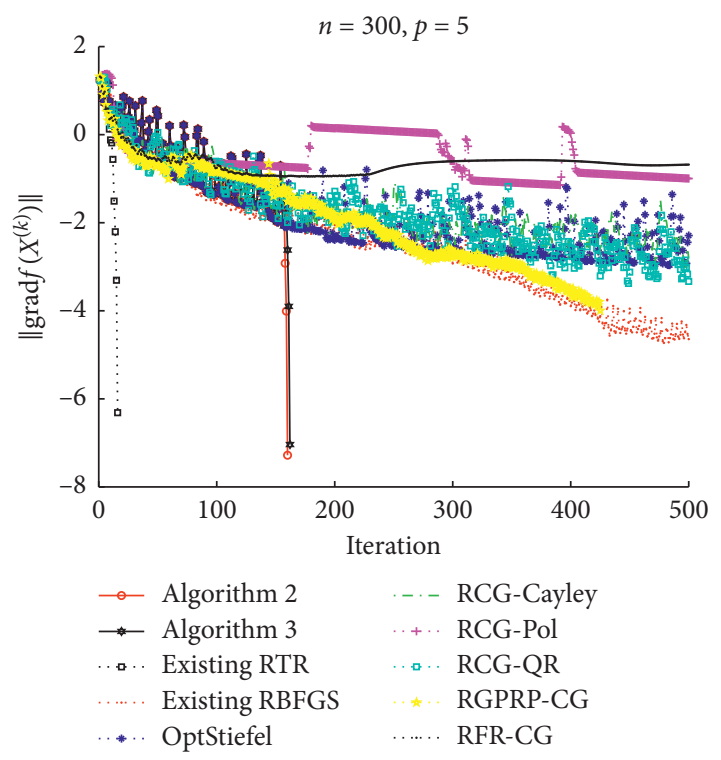

(d)

FIGURE 4: Convergence histories of the considered algorithms with different problem sizes $(n, p)$.

where $W_{\xi^{(k)}}=P_{\xi^{(k)}}^{T} Q_{\xi^{(k)}}$, in which $P_{\xi^{(k)}}=\left[\Pi_{X^{(k)}} \xi^{(k)}\right.$, $\left.X^{(k)}\right]$ and $Q_{\xi^{(k)}}=\left[X^{(k)},-\Pi_{X^{(k)}} \xi^{(k)} \xi^{(k)}\right.$ with $\Pi_{X^{(k)}}=$ $I_{p}-(1 / 2) X^{(k)} X^{(k)^{T}}$. The step size $t_{k}$ is determined by the following nonmonotone line search condition instead of the Wolfe conditions:

$$
\begin{aligned}
f\left(\mathscr{R}_{X^{(k)}}\left(t_{k} \xi^{(k)}\right)\right) \leq & \max \left\{f\left(X^{(k)}\right), \ldots, f\left(X^{\left(k-h_{k}\right)}\right)\right\} \\
& +\delta t_{k}\left\langle\operatorname{grad} f\left(X^{(k)}\right), \xi^{(k)}\right\rangle
\end{aligned}
$$



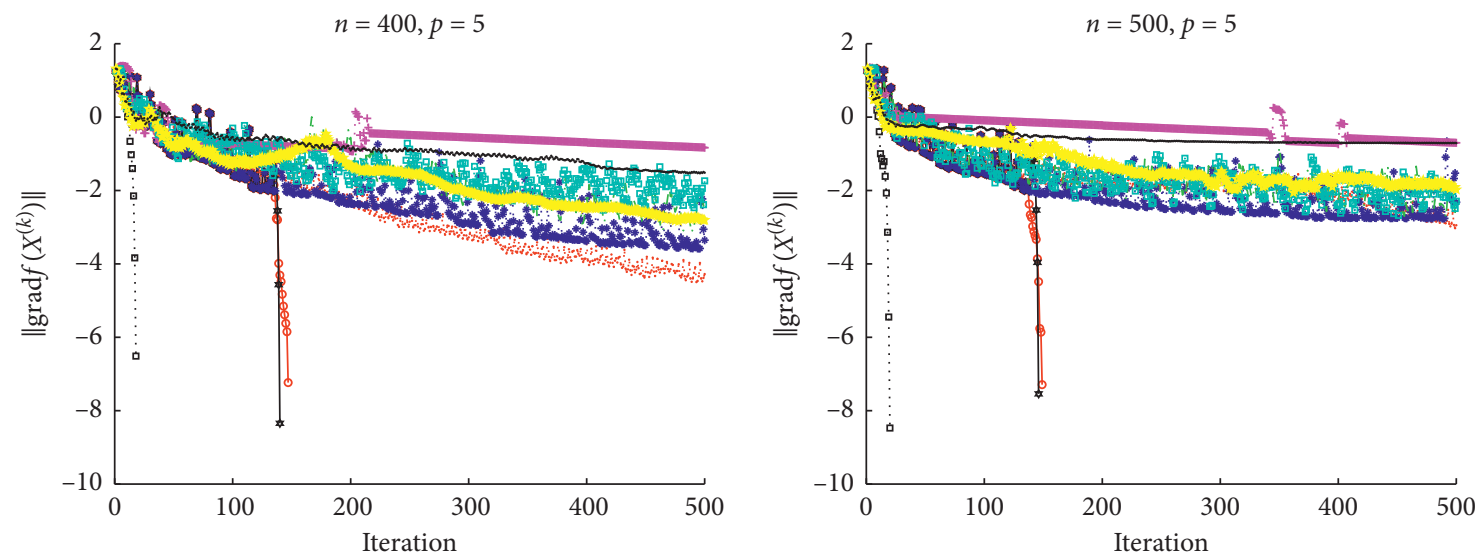

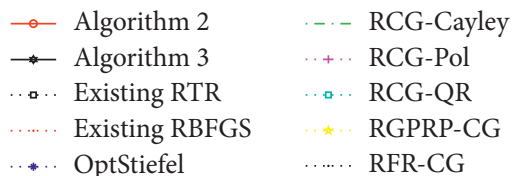

(a)

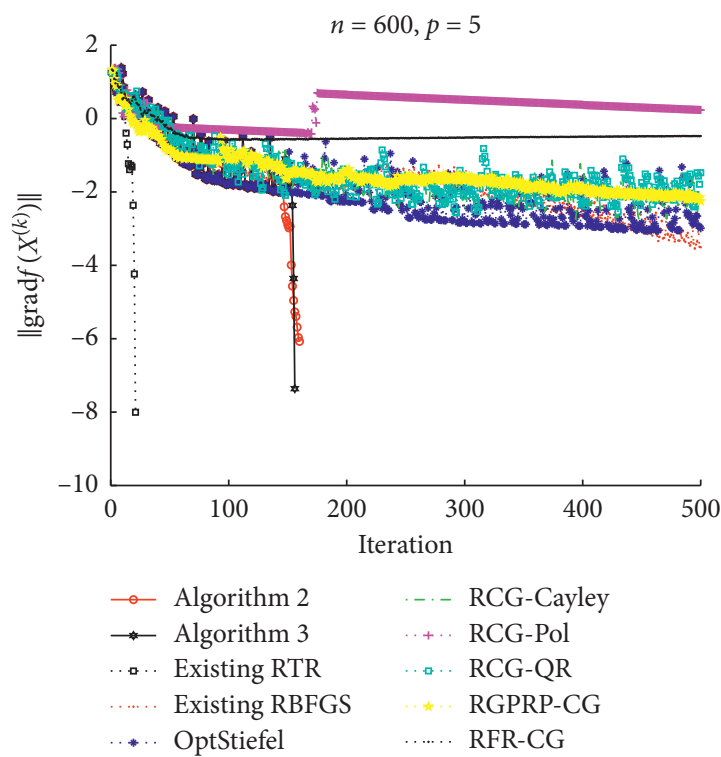

(c)

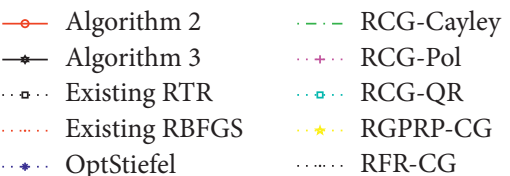

(b)

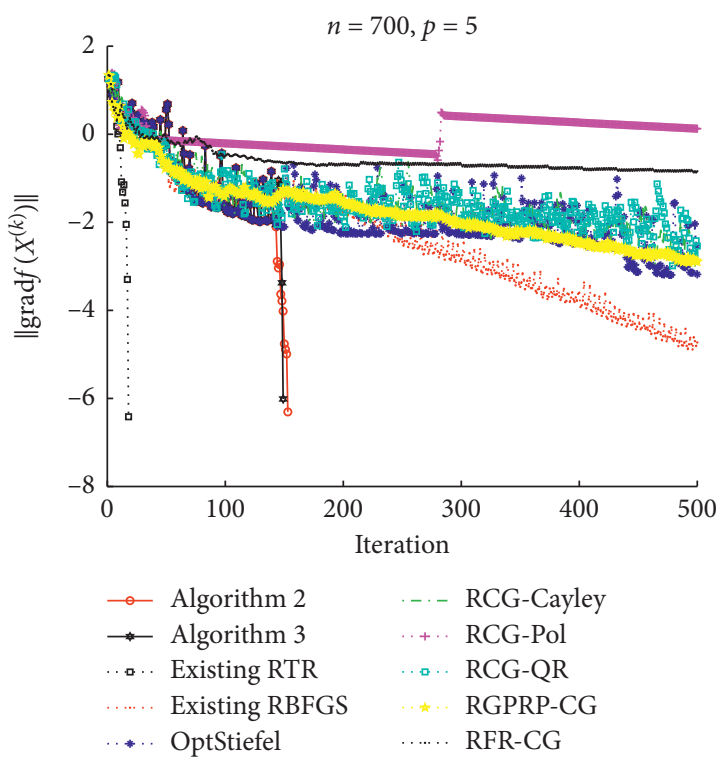

(d)

Figure 5: Convergence histories of the considered algorithms with different problem sizes $(n, p)$.

where $h_{k}=\min \{h-1, k\}$ and $h$ is some positive integer. The parameter $\beta_{k+1}$ is computed as $\beta_{k+1} \in\left[0, \beta_{k+1}^{D}\right]$ with

$$
\beta_{k+1}^{D}=\frac{\left\|\operatorname{grad} f\left(X^{(k+1)}\right)\right\|_{F}^{2}}{\max \left\{\left\langle\operatorname{grad} f\left(X^{(k+1)}\right), \mathscr{T}_{t_{k} \xi^{(k)}}\left(\xi^{(k)}\right)\right\rangle-\left\langle\operatorname{grad} f\left(X^{(k)}\right), \xi^{(k)}\right\rangle,-\left\langle\operatorname{grad} f\left(X^{(k)}\right), \xi^{(k)}\right\rangle\right\}} .
$$


(iv) "RCG-QR": a modification of "RCG-Cayley," by using the QR-based retraction and the corresponding differentiated retraction as a vector transport (see p. 173 of [24]).

(v) "RCG-Pol": another modification of "RCG-Cayley" by using the polar decomposition (18) to construct a retraction and naturally using the corresponding differentiated retraction as a vector transport (see $\mathrm{p}$. 173 of [24]).

In our experiments, the index $m=2$. We consider $S_{i}$ to be correlation matrices of the form

$$
\begin{aligned}
& S_{1}=\left(s_{i j}^{1}\right)_{n \times n}= \begin{cases}1, & i=j ; \\
\frac{1}{i+j-1}, & i \neq j,\end{cases} \\
& S_{2}=\left(s_{i j}^{2}\right)_{n \times n}= \begin{cases}1, & i=j ; \\
0.3, & |i-j|=1 ; \\
0, & \text { else. }\end{cases}
\end{aligned}
$$

In this case, the INDSCAL can be seen as simultaneous principal component analysis of several correlation matrices [10]. We use the diagonal matrices $D_{1}=\operatorname{diag}(p, p-1, \ldots$, $1)$ and $D_{2}=0.5 * \operatorname{diag}(1,2, \ldots, p)$. All starting points $X^{(0)}$ were generated randomly by $X^{(0)}=q f(\operatorname{rand}(n, p))$. In particular, similar to $[16,18]$, a reasonable initial step length in the "RGPRR-CG" and "RFR-CG" methods can be set to

$$
\bar{t}=\left|\frac{\left\langle\operatorname{grad} f\left(X^{(k)}\right), \xi^{(k)}\right\rangle}{\left\langle\operatorname{Hess} f\left(X^{(k)}\right), \xi^{(k)}\right\rangle}\right| .
$$

All the parameters in the compared Riemannian CG methods are chosen in a standard way as in their corresponding literature.

Figures 4 and 5 show the convergence histories of the considered algorithms with different problem sizes $(n, p)$. The number of iterations is plotted on the horizontal axis, and the $\log 10$ of norm of the Riemannian gradient grad $f\left(X^{(k)}\right)$ is plotted on the vertical axis in these figures. The convergence curves generated by Algorithm 2 or Algorithm 3 are composed of two pieces, one of which is an initial part of the curve generated by OptStiefelGBB, and the second piece is the vertical line segment generated by Newton's method. These curves show that the switching of OptStiefel to Newton's one or trust region's one accelerates drastically the speed of convergence. The convergence curve of OptStiefel shows that the convergence of the sequence generated by the OptStiefel is very slow. If the sequence generating method is switched to Newton's method or trustregion method after the iterate $X^{(k)}$ gets into the $\varepsilon_{1}$-neighborhood of the target, the generated sequence reaches, in a few steps, the final point within the required accuracy. More numerical results are reported in Table 5, where the term "IT." in Algorithms 2 and 3 is the same as

\begin{tabular}{|c|c|c|c|c|}
\hline & CT. & IT. & Grad. & Obj. \\
\hline \multicolumn{5}{|l|}{$n=50, p=5$} \\
\hline Algorithm 2 & 0.04 & $127 / 2$ & $6.49 \times 10^{-7}$ & -73.76 \\
\hline Algorithm 3 & 0.35 & $127 / 3$ & $6.41 \times 10^{-11}$ & -73.76 \\
\hline Existing-RTR & 0.44 & 16 & $1.28 \times 10^{-9}$ & -73.76 \\
\hline Existing-RBFGS & 0.87 & 281 & $8.93 \times 10^{-7}$ & -73.76 \\
\hline OptStiefel & 0.04 & 270 & $1.27 \times 10^{-4}$ & -73.76 \\
\hline RCG-Cayley & 0.11 & 446 & $1.83 \times 10^{-4}$ & -73.76 \\
\hline RCG-Pol & 0.84 & 2000 & $7.87 \times 10^{-1}$ & -73.75 \\
\hline RCG-QR & 0.07 & 369 & $1.20 \times 10^{-4}$ & -73.76 \\
\hline RGPRP-CG & 0.04 & 233 & $1.45 \times 10^{-4}$ & -73.76 \\
\hline RFR-CG & 0.29 & 2000 & $1.32 \times 10^{-3}$ & -73.76 \\
\hline \multicolumn{5}{|l|}{$n=100, p=5$} \\
\hline Algorithm 2 & 0.19 & $140 / 4$ & $9.86 \times 10^{-9}$ & -76.97 \\
\hline Algorithm 3 & 0.63 & $140 / 5$ & $5.89 \times 10^{-11}$ & -76.97 \\
\hline Existing-RTR & 0.57 & 15 & $2.95 e-10$ & -76.97 \\
\hline Existing-RBFGS & 1.31 & 387 & $8.63 \times 10^{-7}$ & -76.97 \\
\hline OptStiefel & 0.19 & 501 & $1.26 \times 10^{-4}$ & -76.97 \\
\hline RCG-Cayley & 0.60 & 985 & $2.05 \times 10^{-4}$ & -76.97 \\
\hline RCG-Pol & 2.99 & 2000 & $1.64 \times 10^{-1}$ & -76.95 \\
\hline RCG-QR & 0.43 & 696 & $6.43 \times 10^{-4}$ & -76.97 \\
\hline RGPRP-CG & 0.15 & 353 & $1.32 \times 10^{-4}$ & -76.97 \\
\hline RFR-CG & 0.93 & 2000 & $3.93 \times 10^{-1}$ & -76.87 \\
\hline \multicolumn{5}{|l|}{$n=200, p=5$} \\
\hline Algorithm 2 & 1.74 & $258 / 4$ & $5.72 \times 10^{-8}$ & -79.90 \\
\hline Algorithm 3 & 1.15 & $258 / 5$ & $7.54 \times 10^{-11}$ & -79.90 \\
\hline Existing-RTR & 0.82 & 18 & $3.29 \times 10^{-7}$ & -79.90 \\
\hline Existing-RBFGS & 2.96 & 676 & $8.33 \times 10^{-7}$ & -79.90 \\
\hline OptStiefel & 0.57 & 648 & $1.11 \times 10^{-4}$ & -79.90 \\
\hline RCG-Cayley & 1.45 & 1038 & $7.97 \times 10^{-5}$ & -79.90 \\
\hline RCG-Pol & 5.70 & 1604 & $7.51 \times 10^{-2}$ & -79.87 \\
\hline RCG-QR & 1.33 & 965 & $3.60 \times 10^{-4}$ & -79.90 \\
\hline RGPRP-CG & 0.49 & 511 & $1.46 \times 10^{-4}$ & -79.90 \\
\hline RFR-CG & 1.99 & 2000 & $5.32 \times 10^{-2}$ & -79.90 \\
\hline \multicolumn{5}{|l|}{$n=300, p=5$} \\
\hline Algorithm 2 & 4.60 & $157 / 4$ & $5.25 \times 10^{-8}$ & -81.55 \\
\hline Algorithm 3 & 1.55 & $157 / 5$ & $9.16 \times 10^{-8}$ & -81.55 \\
\hline Existing-RTR & 1.06 & 15 & $4.85 \times 10^{-7}$ & -81.55 \\
\hline Existing-RBFGS & 3.58 & 650 & $8.95 \times 10^{-7}$ & -81.55 \\
\hline OptStiefel & 1.77 & 992 & $1.36 \times 10^{-4}$ & -81.55 \\
\hline RCG-Cayley & 2.09 & 749 & $2.03 \times 10^{-4}$ & -81.55 \\
\hline RCG-Pol & 15.14 & 2000 & $1.55 \times 10^{-2}$ & -81.54 \\
\hline RCG-QR & 1.95 & 703 & $1.34 \times 10^{-4}$ & -81.55 \\
\hline RGPRP-CG & 0.83 & 426 & $1.51 \times 10^{-4}$ & -81.55 \\
\hline RFR-CG & 3.87 & 2000 & $1.74 \times 10^{-1}$ & -81.48 \\
\hline \multicolumn{5}{|l|}{$n=400, p=5$} \\
\hline Algorithm 2 & 21.52 & $135 / 13$ & $5.83 \times 10^{-8}$ & -82.68 \\
\hline Algorithm 3 & 2.59 & $135 / 5$ & $4.53 \times 10^{-9}$ & -82.69 \\
\hline Existing-RTR & 1.41 & 17 & $3.07 \times 10^{-7}$ & -82.69 \\
\hline Existing-RBFGS & 8.27 & 703 & $9.69 \times 10^{-7}$ & -82.69 \\
\hline OptStiefel & 3.41 & 529 & $2.76 \times 10^{-4}$ & -82.69 \\
\hline RCG-Cayley & 8.21 & 787 & $1.61 \times 10^{-4}$ & -82.69 \\
\hline RCG-Pol & 58.01 & 2000 & $6.95 \times 10^{-2}$ & -82.67 \\
\hline RCG-QR & 14.58 & 1420 & $1.15 \times 10^{-3}$ & -82.69 \\
\hline RGPRP-CG & 4.19 & 626 & $1.64 \times 10^{-4}$ & -82.69 \\
\hline RFR-CG & 15.72 & 2000 & $1.63 \times 10^{-1}$ & -82.65 \\
\hline \multicolumn{5}{|l|}{$n=500, p=5$} \\
\hline Algorithm 2 & 28.07 & $138 / 12$ & $5.12 \times 10^{-8}$ & -83.55 \\
\hline Algorithm 3 & 5.40 & $138 / 8$ & $2.87 \times 10^{-8}$ & -83.55 \\
\hline Existing-RTR & 4.09 & 19 & $3.37 \times 10^{-9}$ & -83.55 \\
\hline Existing-RBFGS & 15.39 & 917 & $1.00 \times 10^{-6}$ & -83.55 \\
\hline
\end{tabular}
that in Table 3 and "Obj.," means the objective function
TABLe 5: Comparison results of the considered algorithms. 
TABle 5: Continued.

\begin{tabular}{lcccc}
\hline & CT. & IT. & Grad. & Obj. \\
\hline OptStiefel & 13.00 & 1317 & $1.66 \times 10^{-4}$ & -83.55 \\
RCG-Cayley & 29.72 & 1829 & $2.70 \times 10^{-4}$ & -83.55 \\
RCG-Pol & 82.85 & 1838 & $3.66 \times 10^{-2}$ & -83.54 \\
RCG-QR & 24.98 & 1569 & $4.00 \times 10^{-4}$ & -83.55 \\
RGPRP-CG & 11.26 & 1051 & $1.22 \times 10^{-4}$ & -83.55 \\
RFR-CG & 21.09 & 2000 & $1.88 \times 10^{-1}$ & -83.48 \\
$n=600, p=5$ & & & & \\
Algorithm 2 & 56.49 & $147 / 14$ & $8.44 \times 10^{-7}$ & -84.25 \\
Algorithm 3 & 6.28 & $147 / 9$ & $4.36 \times 10^{-8}$ & -84.25 \\
Existing-RTR & 2.97 & 20 & $9.91 \times 10^{-9}$ & -84.25 \\
Existing-RBFGS & 16.56 & 800 & $9.75 \times 10^{-7}$ & -84.25 \\
OptStiefel & 20.03 & 1436 & $1.28 \times 10^{-4}$ & -84.25 \\
RCG-Cayley & 32.21 & 1427 & $2.60 \times 10^{-4}$ & -84.25 \\
RCG-Pol & 125.53 & 2000 & $3.98 \times 10^{-1}$ & -84.24 \\
RCG-QR & 24.08 & 1067 & $2.53 \times 10^{-4}$ & -84.25 \\
RGPRP-CG & 17.47 & 1150 & $1.47 \times 10^{-4}$ & -84.25 \\
RFR-CG & 30.46 & 2000 & $4.96 \times 10^{-1}$ & -84.12 \\
\hline$n=700, p=5$ & & & & \\
Algorithm 2 & 67.50 & $143 / 11$ & $4.94 \times 10^{-7}$ & -84.83 \\
Algorithm 3 & 5.08 & $143 / 6$ & $9.72 \times 10^{-7}$ & -84.84 \\
Existing-RTR & 3.36 & 17 & $3.83 \times 10^{-7}$ & -84.84 \\
Existing-RBFGS & 16.68 & 609 & $1.04 \times 10^{-6}$ & -84.84 \\
OptStiefel & 13.63 & 720 & $1.22 \times 10^{-4}$ & -84.84 \\
RCG-Cayley & 25.07 & 839 & $1.57 \times 10^{-4}$ & -84.84 \\
RCG-Pol & 171.96 & 2000 & $1.53 \times 10^{-1}$ & -84.82 \\
RCG-QR & 22.82 & 793 & $4.87 \times 10^{-5}$ & -84.84 \\
RGPRP-CG & 14.48 & 717 & $1.35 \times 10^{-4}$ & -84.84 \\
RFR-CG & 41.21 & 2000 & $9.98 \times 10^{-2}$ & -84.81 \\
\hline & & & &
\end{tabular}

value $-2 \sum_{j=1}^{m} \operatorname{tr}\left(S_{j} X^{(k)} D_{j} X^{(k)^{T}}\right)$ at the final iterate. We can observe from Table 5 that Algorithm 3, with the implementation of OptStiefel to generate a suitable initial point, works very effectively for the orthonormal INDSCAL fitting problem and always performs much better than the existing Riemannian BFGS algorithm and all the compared Riemannian gradient-types methods in terms of total computing time and the accuracy of the solution. On the other hand, we note that the OptStiefel part of Algorithm 3 seems to be like preconditioning. In most cases, the preconditioning scheme can bring a significant reduction in terms of iteration steps than the existing Riemannian RTR method.

We can also see from Table 5 that when the problem size is large, Algorithm 2 uses much more computing time than that of other methods. This is because Newton's method involve an inner iteration, the CR method to the symmetric linear equation (69), it consumes a lot of time to get an approximate solution to the equation, especially when the system dimension is large. Even though Newton's method takes the longest time per iteration among the considered algorithms, the convergence is very quick as a whole.

\section{Conclusion}

We have dealt with the trace function minimization problem with orthogonal constraints as an optimization problem on the manifold $\mathrm{St}(n, p)$ and have developed Riemannian Newton's method and Riemannian trust-region method for the problem. For diminishing the difficulty in solving Newton's equation in Newton's method, we have computed the representation matrix of the Hessian of the objective function using the vectorization operators and have reduced Newton's equation into a standard symmetric linear equation with dimension reduction. In addition, we have proposed a new trust-region method in which trust-region subproblems are solved by the truncated conjugate gradient method based on our expressions of the Hessian of the objective function. Furthermore, we have performed numerical experiments to verify that our present algorithms almost always perform much better than some Riemannian gradient-type methods.

\section{Data Availability}

The data used to support the findings of this study are included within the article.

\section{Conflicts of Interest}

The authors declare that they have no conflicts of interest.

\section{Acknowledgments}

Part of this work was done when the first author was visiting the Department of Mathematics, Southern Illinois University Carbondale. He is grateful for providing good working conditions. The authors thank Prof. Mingqing Xiao for discussing the practical application of Problem 1 in multivariate statistics. This research was supported by the National Natural Science Foundation of China (11761024, 11561015, and 11961012), the Natural Science Foundation of Guangxi Province (2016GXNSFAA380074, 2016GXNSFFA380009, and 2017GXNSF-BA198082), the GUET Excellent Graduate Thesis Program (17YJPYSS24 and 2019YJSPY03), and the GUET Graduate Innovation Project (2020YJSCX02).

\section{References}

[1] H. A. L. Kiers, "Majorization as a tool for optimizing a class of matrix functions," Psychometrika, vol. 55, no. 3, pp. 417-428, 1990.

[2] H. A. L. Kiers and J. M. F. ten Berge, "Minimization of a class of matrix trace functions by means of refined majorization," Psychometrika, vol. 57, no. 3, pp. 371-382, 1992.

[3] H. A. L. Kiers, "Setting up alternating least squares and iterative majorization algorithms for solving various matrix optimization problems," Computational Statistics \& Data Analysis, vol. 41, no. 1, pp. 157-170, 2002.

[4] C. Bijleveld and J. de Leeuw, "Fitting linear dynamical systems by alternating least squares," in Proceedings of the 1987 European Meeting of the Psychometric Society, Twente, Netherlands, 1987.

[5] R. A. Harshman, "Models for analysis of asymmetrical relationships among $\mathrm{N}$ objects or stimuli," in Proceedings of the 1st Joint Meeting of the Psychometric Society and the Society of Mathematical Psychology, Hamilton, Ontario, 1978.

[6] H. A. Kiers, J. M. ten Berge, Y. Takane, and J. de Leeuw, "A generalization of Takane's algorithm for DEDICOM," Psychometrika, vol. 55, no. 1, pp. 151-158, 1990. 
[7] P. M. Kroonenberg, Three-Mode Principal Component Analysis: Theory and Applications, Vol. 2, DSWO Press, Leiden, Netherlands, 1983.

[8] J. T. Berge, "A treatment of the orthomax rotation family in terms of diagonalization, and a re-examination of a singular value approach to varimax rotation," Computational Statistics Quarterly, vol. 3, pp. 207-217, 1988.

[9] H. A. Kiers and J. M. ten Berge, "Hierarchical relations between methods for simultaneous component analysis and a technique for rotation to a simple simultaneous structure," British Journal of Mathematical and Statistical Psychology, vol. 47, no. 1, pp. 109-126, 1994.

[10] N. T. Trendafilov, "Orthonormality-constrained INDSCAL with nonnegative saliences," in Proceedings of the 2004 International Conference on Computational Science and Its Applications, pp. 952-960, Assisi, Italy, May 2004.

[11] J. Nocedal and S. Wright, Numerical Optimization, Springer Science \& Business Media, Berlin, Germany, 2006.

[12] T. E. Abrudan, J. Eriksson, and V. Koivunen, "Steepest descent algorithms for optimization under unitary matrix constraint," IEEE Transactions on Signal Processing, vol. 56, no. 3, pp. 1134-1147, 2008.

[13] Z. Wen and W. Yin, "A feasible method for optimization with orthogonality constraints," Mathematical Programming, vol. 142, no. 1-2, pp. 397-434, 2013.

[14] X. Zhu, "A Riemannian conjugate gradient method for optimization on the Stiefel manifold," Computational Optimization and Applications, vol. 67, no. 1, pp. 73-110, 2017.

[15] J. F. Li, W. Li, S. W. Vong, Q. L. Luo, and M. Xiao, "A Riemannian optimization approach for solving the generalized eigenvalue problem for nonsquare matrix pencils," Journal of Scientific Computing, vol. 82, no. 3, pp. 1-43, 2020.

[16] Z. Zhao, X. Q. Jin, and Z. J. Bai, "A geometric nonlinear conjugate gradient method for stochastic inverse eigenvalue problems," SIAM Journal on Numerical Analysis, vol. 54, no. 4, pp. 2015-2035, 2016.

[17] H. Sato and T. Iwai, "A Riemannian optimization approach to the matrix singular value decomposition," SIAM Journal on Optimization, vol. 23, no. 1, pp. 188-212, 2013.

[18] T. T. Yao, Z. J. Bai, Z. Zhao, and W. K. Ching, "A Riemannian Fletcher-Reeves conjugate gradient method for doubly stochastic inverse eigenvalue problems," SIAM Journal on Matrix Analysis and Applications, vol. 37, no. 1, pp. 215-234, 2016.

[19] D. Gabay, "Minimizing a differentiable function over a differential manifold," Journal of Optimization Theory and Applications, vol. 37, no. 2, pp. 177-219, 1982.

[20] A. Edelman, T. A. Arias, and S. T. Smith, "The geometry of algorithms with orthogonality constraints," SIAM Journal on Matrix Analysis and Applications, vol. 20, no. 2, pp. 303-353, 1998.

[21] K. Aihara and H. Sato, "A matrix-free implementation of Riemannian Newton's method on the Stiefel manifold," Optimization Letters, vol. 11, no. 8, pp. 1729-1741, 2017.

[22] H. Sato, "Riemannian Newton-type methods for joint diagonalization on the Stiefel manifold with application to independent component analysis," Optimization, vol. 66, no. 12, pp. 2211-2231, 2017.

[23] J. Hu, A. Milzarek, Z. Wen, and Y. Yuan, "Adaptive quadratically regularized Newton method for Riemannian optimization," SIAM Journal on Matrix Analysis and Applications, vol. 39, no. 3, pp. 1181-1207, 2018.

[24] P. A. Absil, R. Mahony, and R. Sepulchre, Optimization Algorithms on Matrix Manifolds, Princeton University Press, Princeton, NJ, USA, 2009.
[25] P. A. Absil, C. G. Baker, and K. A. Gallivan, "Trust-region methods on Riemannian manifolds with applications in numerical linear algebra," in Proceedings of the 16th International Symposium on Mathematical Theory of Networks and Systems (MTNS2004), Leuven, Belgium, 2004.

[26] C. G. Baker, P. A. Absil, and K. A. Gallivan, "An implicit trustregion method on Riemannian manifolds," IMA Journal of Numerical Analysis, vol. 28, no. 4, pp. 665-689, 2008.

[27] J. Hu, X. Liu, Z. Wen, and Y. Yuan, "A brief introduction to manifold optimization," 2019, https://arxiv.org/abs/1906. 05450.

[28] L. H. Zhang, "Riemannian trust-region method for the maximal correlation problem," Numerical Functional Analysis and Optimization, vol. 33, no. 3, pp. 338-362, 2012.

[29] P. Yang, Y. L. Jiang, and K. L. Xu, "A trust-region method for $\mathrm{H} 2$ model reduction of bilinear systems on the Stiefel manifold," Journal of the Franklin Institute, vol. 356, no. 4, pp. 2258-2273, 2019.

[30] H. Sato, "Joint singular value decomposition algorithm based on the Riemannian trust-region method," JSIAM Letters, vol. 7, pp. 13-16, 2015.

[31] Y. Saad, Iterative Methods for Sparse Linear Systems, Vol. 82, SIAM, Philadelphia, PA, USA, 2003.

[32] P. A. Absil, R. Mahony, and J. Trumpf, "An extrinsic look at the Riemannian Hessian," in Proceedings of the 2013 International Conference on Geometric Science of Information, pp. 361-368, Paris, France, 2013.

[33] H. V. Henderson and S. R. Searle, "The vec-permutation matrix, the vec operator and Kronecker products: a review," Linear and Multilinear Algebra, vol. 9, no. 4, pp. 271-288, 1981.

[34] O. P. Ferreira and R. C. Silva, "Local convergence of Newton's method under a majorant condition in Riemannian manifolds," IMA Journal of Numerical Analysis, vol. 32, no. 4, pp. 1696-1713, 2012.

[35] J. Barzilai and J. M. Borwein, "Two-point step size gradient methods," IMA Journal of Numerical Analysis, vol. 8, no. 1, pp. 141-148, 1988.

[36] H. Zhang and W. W. Hager, "A nonmonotone line search technique and its application to unconstrained optimization," SIAM Journal on Optimization, vol. 14, no. 4, pp. 1043-1056, 2004.

[37] P. A. Absil, C. G. Baker, and K. A. Gallivan, "Trust-region methods on Riemannian manifolds," Foundations of Computational Mathematics, vol. 7, no. 3, pp. 303-330, 2007.

[38] N. Boumal, B. Mishra, P. A. Absil, and R. Sepulchre, "Manopt, a Matlab toolbox for optimization on manifolds," Journal of Machine Learning Research, vol. 15, no. 1, pp. 1455-1459, 2014. 